\title{
OVERCONVERGENT QUATERNIONIC FORMS AND ANTICYCLOTOMIC $p$-ADIC $L$-FUNCTIONS
}

\author{
Chan-Ho KIM
}

\begin{abstract}
We reinterpret the explicit construction of Gross points given by ChidaHsieh as a non-Archimedian analogue of the standard geodesic cycle $(i \infty)-(0)$ on the Poincaré upper half plane. This analogy allows us to consider certain distributions, which can be regarded as anticyclotomic $p$-adic $L$-functions for modular forms of non-critical slope following the overconvergent strategy à la Stevens. We also give a geometric interpretation of their Gross points for the case of weight two forms. Our construction generalizes those of Bertolini-Darmon, Bertolini-Darmon-Iovita-Spiess, and Chida-Hsieh and shows a certain integrality of the interpolation formula even for non-ordinary forms.
\end{abstract}

2010 Mathematics Subject Classification: Priimary: 11R23; Secondary: 11F33.

Key words: Iwasawa theory, $p$-adic $L$-functions, Gross points, quaternion algebras, automorphic forms.

\section{Contents}

1. Introduction

2. Explicit Gross points à la Chida and Hsieh

3. Geometric Gross points for weight two forms

4. Comparison among Gross points

5. Coefficients

6. Overconvergent quaternionic forms and control theorems

7. Construction of $p$-adic $L$-functions

8. The "weak" interpolation formula

9. Speculations and questions 


\section{Introduction}

1.1. Overview. It is now widely known that "overconvergent methods" yield simpler and more algorithmically efficient constructions of $p$-adic $L$-functions [Ste], [PS1] and conjectural algebraic points of elliptic curves, so called Darmon-Stark-Heegner points $[\mathbf{D P}],[\mathbf{G M}]$. In [Ste], Stevens provides a simple and beautiful construction of Mazur-TateTeitelbaum $p$-adic $L$-functions of modular forms under cyclotomic extensions using distribution-valued modular symbols. In [GS], measurevalued modular symbols, which can be regarded as a special case, are used in the proof of the exceptional zero conjecture [MTT, $\S 15, \S 16]$ as an essential ingredient.

In this article we apply Stevens' "overconvergent" idea to the anticyclotomic setting. Instead of using modular symbols, we use automorphic forms on a definite quaternion algebra (quaternionic forms, for short). Although modular symbols and quaternionic forms have certain similarities in their shape, their domains are fundamentally different. Modular symbols are essentially defined on the upper half plane, which lies in the complex world, and quaternionic forms are defined on the double coset space arising from the quaternion algebra. Note that, in the case of weight two forms, it can be realized in terms of the Bruhat-Tits tree for $\mathrm{PGL}_{2}\left(\mathbb{Q}_{p}\right)$ or its variant, which lies in the $p$-adic world.

Using the theory of overconvergent modular symbols, it is proved that the evaluation of the overconvergent modular symbol attached to a non-critical slope eigenform at the cycle $(i \infty)-(0)$ on the upper half plane gives us the $p$-adic distribution corresponding to the Mazur-TateTeitelbaum $p$-adic $L$-function of the form.

We develop an analogous theory for overconvergent quaternionic forms. Since the domain is fundamentally different from the case of modular symbols, we naturally meet the following question.

Question 1.1. What is an analogue of the geodesic cycle $(i \infty)-(0) \in$ $\operatorname{Div}^{0}\left(\mathbb{P}^{1}(\mathbb{Q})\right)$ in the quaternionic setting?

The main contribution of this article is to provide an answer to this question by taking the full advantage of the explicit construction of Gross points à la Chida-Hsieh. We will call such an analogue the explicit Gross point. Also, in the case of weight two forms, we give another interpretation of these points in terms of the Bruhat-Tits tree for $\mathrm{PGL}_{2}\left(\mathbb{Q}_{p}\right)$.

As an application of these points, we are able to generalize the construction of anticyclotomic $p$-adic $L$-functions to modular forms of noncritical slope and to prove an integrally refined interpolation formula 
for those forms. Our construction generalizes those in [BD1], [BDIS], and $[\mathbf{C H}]$. Comparing with various automorphic constructions of $p$ adic $L$-functions including the works of Deppe [Dep], Bergunde-Gehrmann [BG], and Molina [Mol], our construction has advantages due to its explicit nature. Our construction allows an integrally refined interpolation formula even for modular forms of non-critical slope (with moderate growth). See Corollary 8.9 and Remark 8.10. Those automorphic constructions work only for ordinary automorphic forms of weight two although they extend in different directions (e.g. $\mathrm{GL}_{2}$ over totally real or number fields). Also, for the computational aspect, we expect that an efficient algorithm to compute anticyclotomic $p$-adic $L$-functions can be implemented via our overconvergent construction. See $[\mathbf{D H H}+]$ for the cyclotomic case.

For the construction, we recall the notion of overconvergent quaternionic forms and (re)prove the control theorem for overconvergent quaternionic forms of non-critical slope. This generalizes [LV2, §3], which deals with the control theorem for the slope zero subspace. Also, our approach yields a certain integrality of the control theorem for the slope zero subspace. The reader can easily observe that we care for the integrality of $L$-values and automorphic forms even for the non-ordinary case. Note that the theory of overconvergent quaternionic forms has many arithmetic applications including the factorization of Hida $p$-adic $L$-functions [BD6] and Teitelbaum $L$-invariants [BDI].

We expect that the explicit Gross points can be reinterpreted as a functional on the completed cohomology for quaternion algebras sending cuspidal eigenforms to (one half of) their anticyclotomic $p$-adic $L$-functions.

In the sequel paper in preparation we construct integral anticyclotomic $p$-adic $L$-functions for Hida families, which are two variable ones, and prove the vanishing of $\mu$-invariant of each member of the families under mild assumptions, generalizing $[\mathbf{C K L}]$. In $[\mathbf{C K L}]$ a different approach was taken following $[\mathbf{L V} \mathbf{1}]$ and $[\mathbf{C L}]$ using compatible families of Gross points in the tower of Gross curves, so called big Gross points. Note that the approach using big Gross points does not work for the non-ordinary case. 
The following diagram describes the flowchart for the classical constructions of cyclotomic and anticyclotomic $p$-adic $L$-functions of modular forms. The upper (= cyclotomic) part of the diagram is well-documented in [Pol2]:

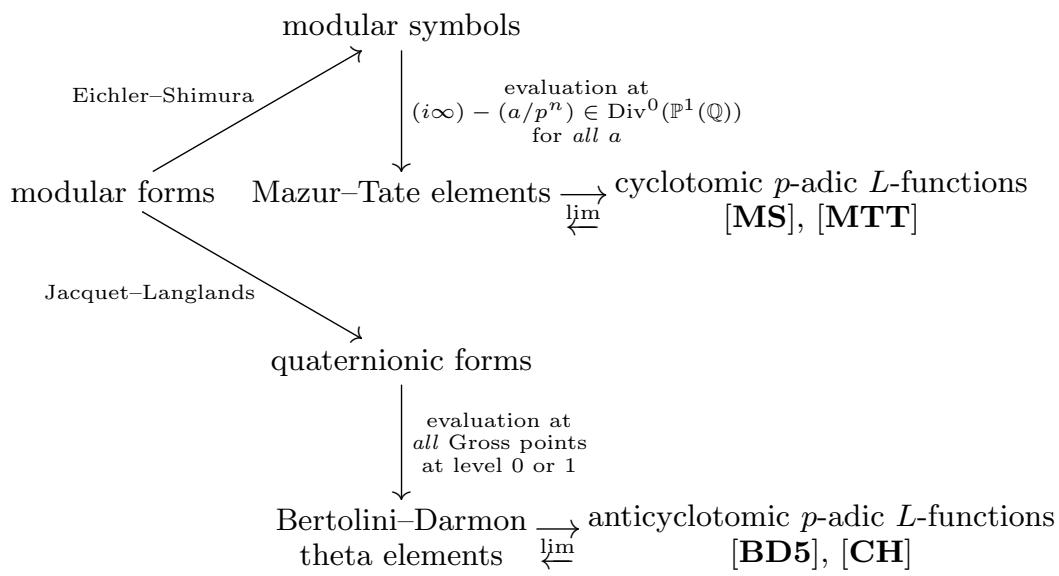

The overconvergent method shows that it suffices to evaluate overconvergent modular symbols or overconvergent quaternionic forms "at one point". This is because the overconvergent method pushes the complexity of the evaluation of classical quaternionic forms at all Gross points (§3.2) into the complexity of the coefficient modules (the distribution modules) of overconvergent quaternionic forms ( $\$ 5.2$ and $\S 7.1)$. The bold part of the following diagram is the main content of this article:

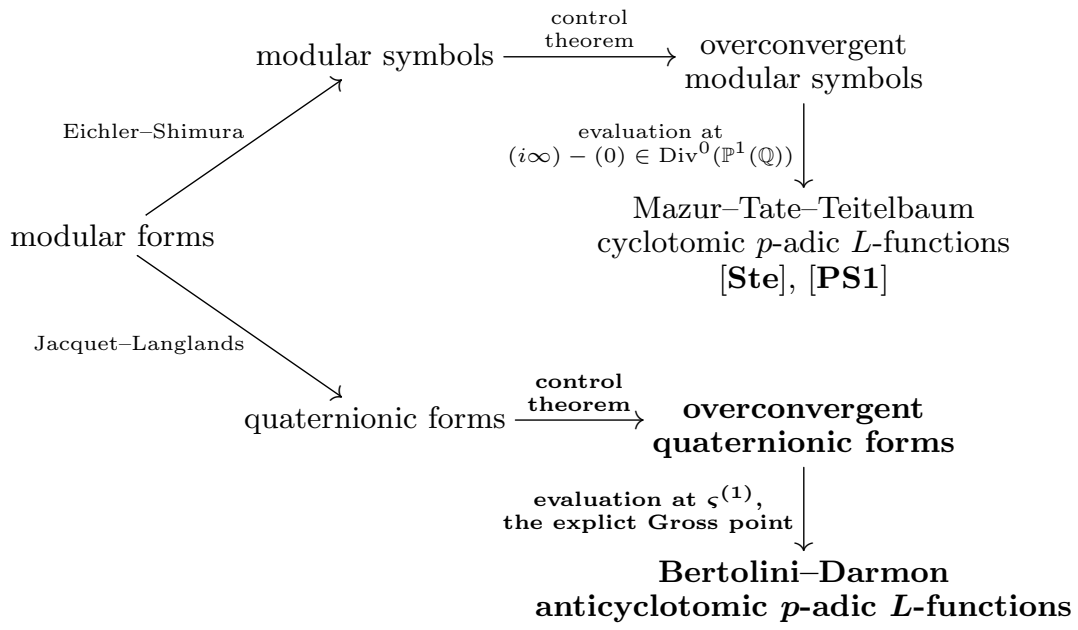


1.2. Setting the basic stage. Let $p$ be a prime $\geq 3$ and $k \in \mathbb{Z}_{\geq 2}$. Fix an algebraic closure $\overline{\mathbb{Q}}$ of $\mathbb{Q}$ and embeddings $\iota_{\infty}: \overline{\mathbb{Q}} \hookrightarrow \mathbb{C}$ and $\iota_{p}: \overline{\mathbb{Q}} \hookrightarrow$ $\mathbb{C}_{p}$. Let $\Gamma_{0}(N)$ be the congruence subgroup of level $N$ with $(N, p)=$ 1. Let $f_{k}=\sum a_{n}\left(f_{k}\right) q^{n} \in S_{k}\left(\Gamma_{0}(N p)\right)$ be a $p$-stabilized newform of slope $h=\operatorname{ord}_{p}\left(\alpha_{p}\left(f_{k}\right)\right)<k-1$ with the convention $\operatorname{ord}_{p}(p)=1$, i.e. the slope of $f$ is non-critical.

Fix an imaginary quadratic field $K$ with $(\operatorname{disc}(K), p N)=1$. The choice of $K$ determines the decomposition of $N$ as follows:

$$
N=N^{+} \cdot N^{-},
$$

where a prime divisor of $N^{+}$splits in $K$ and a prime divisor of $N^{-}$is inert in $K$.

Assumption 1.2. In Equation (1.1) $N^{-}$is square-free and the product of an odd number of primes.

Let $K_{\infty}$ be the anticyclotomic $\mathbb{Z}_{p}$-extension of $K$ and write $\Gamma_{\infty}=$ $\operatorname{Gal}\left(K_{\infty} / K\right) \simeq \mathbb{Z}_{p}$ (non-canonically). Write $K_{n}$ for the unique subfield of $K_{\infty}$ such that $\Gamma_{n}=\operatorname{Gal}\left(K_{n} / K\right) \simeq \mathbb{Z} / p^{n} \mathbb{Z}$.

Let $B$ be the definite quaternion algebra over $\mathbb{Q}$ of discriminant $N^{-}$ and $R=R_{N^{+}}$be an (oriented) Eichler order of level $N^{+}$. For each prime $\ell \nmid N^{-}$, we fix an embedding $R_{\ell}:=R \otimes_{\mathbb{Z}} \mathbb{Z}_{\ell} \hookrightarrow \mathrm{M}_{2}\left(\mathbb{Z}_{\ell}\right)$ and we identify them under this isomorphism. Let $\widehat{A}:=A \otimes_{\mathbb{Z}} \widehat{\mathbb{Z}}$ for any abelian group $A$.

For each $r \geq 0$, let $R_{N^{+} p^{r}}$ be an Eichler order of level $N^{+} p^{r}$ such that

$$
R_{N^{+} p^{r}, p}^{\times}:=\left(R_{N^{+} p^{r}} \otimes_{\mathbb{Z}} \mathbb{Z}_{p}\right)^{\times}=\left\{\left(\begin{array}{ll}
a & b \\
c & d
\end{array}\right) \in \mathrm{GL}_{2}\left(\mathbb{Z}_{p}\right): c \in p^{r} \mathbb{Z}_{p}\right\}
$$

and its prime-to- $p$ component coincides with that of $\widehat{R}^{\times}$. We also write $\Gamma_{0}\left(p^{r} \mathbb{Z}_{p}\right)=R_{N^{+} p^{r}, p}^{\times}$. Note that $\widehat{R}_{N^{+} p^{r}}^{\times}$corresponds to $\Gamma_{0}\left(N^{+} p^{r}\right)$-level structures in the classical sense.

Let $E$ be a finite extension of $\mathbb{Q}_{p}$ large enough to contain all the Hecke eigenvalues of $f_{k}$ and write $\mathcal{O}=\mathcal{O}_{E}$.

Let $\mathscr{D}_{k} / \mathbf{D}_{k}$ be the space of $E$-valued locally/rigid analytic distributions on $\mathbb{Z}_{p}$ with weight $k$ action of a certain semigroup $\Sigma_{0}(p)$, respectively. Let $\mathscr{D}_{k}(\mathcal{O}) / \mathbf{D}_{k}(\mathcal{O})$ be the subspaces of $\mathcal{O}$-valued locally/rigid analytic distributions of $\mathscr{D}_{k} / \mathbf{D}_{k}$, respectively. See $\S 5.2$ for detail.

Let $S_{k}^{N^{-}}\left(N^{+} p, E\right)$ be the space of $E$-valued quaternionic forms of weight $k$, level $N^{+} p$, and discriminant $N^{-}$, and denote its overconvergent variants by $S^{N^{-}}\left(N^{+} p, \mathscr{D}_{k}\right), S^{N^{-}}\left(N^{+} p, \mathbf{D}_{k}\right)$, which are defined in $\S 6$. 
For any Hecke module $S$, let $S^{(<h)}$ be the subspace of $S$ consisting of the members whose slopes are less than $h$, and $S^{(0)}$ be the slope zero subspace.

1.3. A precise formulation of Question 1.1 and its answer. For a cuspidal eigenform $f_{k}$ of non-critical slope, let

$$
\phi_{f_{k}}^{m s}: \operatorname{Div}^{0}\left(\mathbb{P}^{1}(\mathbb{Q})\right) \longrightarrow \operatorname{Sym}^{k-2}\left(\overline{\mathbb{Z}}_{p}^{2}\right)
$$

be the integrally normalized corresponding modular symbol defined using the Eichler-Shimura map. Looking at the diagram

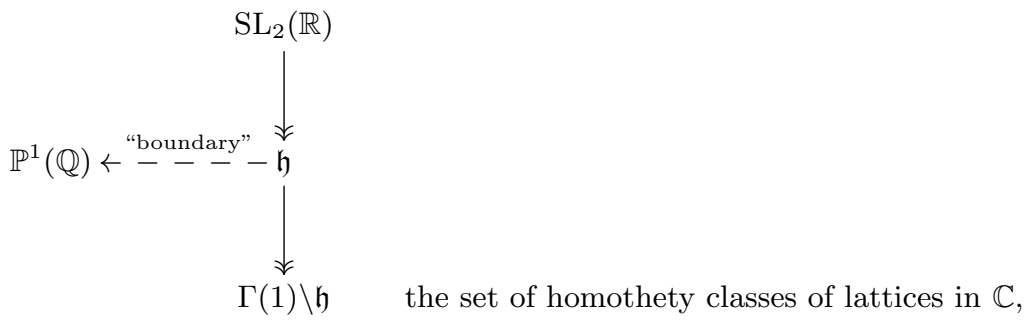

we may consider $\phi_{f_{k}}^{m s}$ as "a function on the upper half plane $\mathfrak{h}$ ", at least intuitively. Indeed, the modular symbols are computed in terms of the period integrals on $\mathfrak{h}$ (as in [Pol2, §2]).

By $\left[\right.$ Ste] (see also [Gre] and [PS1]), we can uniquely lift $\phi_{f_{k}}$ to the overconvergent modular symbol

$$
\Phi_{f_{k}}^{m s}: \operatorname{Div}^{0}\left(\mathbb{P}^{1}(\mathbb{Q})\right) \longrightarrow \mathscr{D}_{k} .
$$

Then the overconvergent modular symbol $\Phi_{f_{k}}^{m s}$ directly yields the MazurTate-Teitelbaum $p$-adic $L$-function as a distribution by

$$
L_{p}\left(\mathbb{Q}\left(\mu_{p} \infty\right), f_{k}\right)=\Phi_{f_{k}}^{m s}((i \infty)-(0)) .
$$

In the anticyclotomic case, certain special points on the adelic double coset space arising from quaternion algebras, called (classical) Gross points, play the same role as $(i \infty)-\left(a / p^{n}\right) \in \operatorname{Div}^{0}\left(\mathbb{P}^{1}(\mathbb{Q})\right)$ for the construction of anticyclotomic $p$-adic $L$-function of weight two ordinary forms. We will review this in $§ 3.2$.

There are several approaches toward the generalization to the higher weight forms including $[$ BDIS $]$, $[\mathbf{B D 6}]$, and $[\mathbf{C H}]$ but with limitations. One of the obstructions is the lack of the "right infinite level space" where the Gross points live. More precisely, the domain of higher weight quaternionic forms lies in a "deeper" level than the domain where the Gross points are canonically defined. 
Mimicking the above picture in the quaternionic setting, we have a slightly more complicated picture:

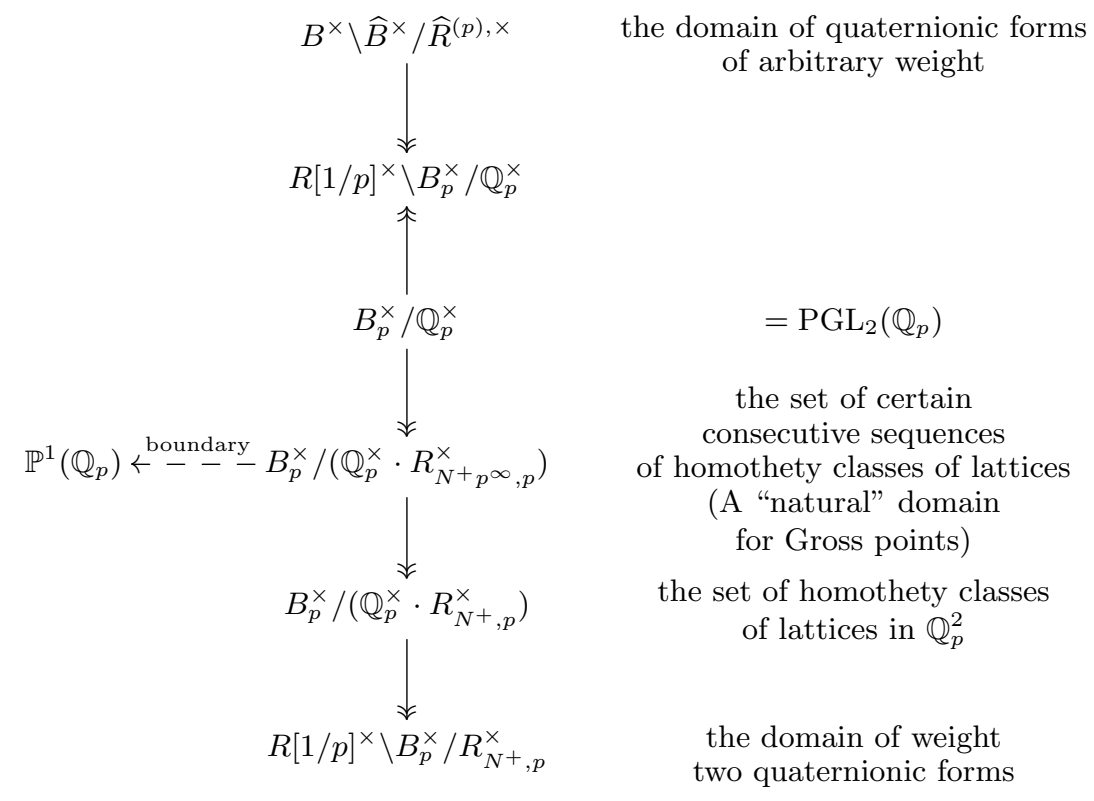

The naïve analogy suggests us considering $B^{\times} \backslash \widehat{B}^{\times} / \widehat{R}_{N^{+} p^{\infty}}^{\times} \simeq B_{p}^{\times} /\left(\mathbb{Q}_{p}^{\times}\right.$. $\left.R_{N^{+} p^{\infty}, p}^{\times}\right)$or even $\mathrm{PGL}_{2}\left(\mathbb{Q}_{p}\right)$ as the domain of the quaternionic forms, but it is not true. Thus, the naïve analogy does not gives us a chance to find an analogous element of $(i \infty)-(0) \in \operatorname{Div}^{0}\left(\mathbb{P}^{1}(\mathbb{Q})\right)$ in $B^{\times} \backslash \widehat{B}^{\times} / \widehat{R}^{(p), \times}$ if the weight of the form $>2$. As in the picture, the domain of quaternionic forms lies "deeper" than $B_{p}^{\times} /\left(\mathbb{Q}_{p}^{\times} \cdot R_{N^{+} p^{\infty}, p}^{\times}\right)$if their weight is $>2$, and even the domain $B^{\times} \backslash \widehat{B}^{\times} / \widehat{R}^{(p), \times}$ has no direct geometric description as far as we know. In the case of weight two forms, it suffices to find (classical) Gross points on $B_{p}^{\times} /\left(\mathbb{Q}_{p}^{\times} \cdot R_{N^{+}, p}^{\times}\right)$; thus, the naïve analogy works well and the classical Gross points can be lifted to geometric Gross points on $B_{p}^{\times} /\left(\mathbb{Q}_{p}^{\times} \cdot R_{N^{+} p^{\infty}, p}^{\times}\right)$. Although it seems difficult to find a geometric motivation, Chida and Hsieh directly and explicitly constructed Gross points on $\widehat{B}^{\times}$in $[\mathbf{C H}]$. Their construction allows us to find the analogue of $(i \infty)-(0)$ for the quaternionic setting. We review their explicit construction of Gross points ("explicit Gross points") in $\S 2$, give them an geometric interpretation for the case of weight two forms ("geometric Gross points") in $\S 3$, and compare these points in $\S 4$. 
1.4. Control theorems. In $\S 6.5$, we reprove the following control theorem for non-critical slope forms.

Theorem 1.3 (Theorem 6.6). There exist Hecke-equivariant isomorphisms

$$
\begin{aligned}
S^{N^{-}}\left(N^{+} p, \mathscr{D}_{k}\right)^{(<k-1)} & \stackrel{\simeq}{\longrightarrow} S^{N^{-}}\left(N^{+} p, \mathbf{D}_{k}\right)^{(<k-1)} \\
& \simeq S_{k}^{N^{-}}\left(N^{+} p, E\right)^{(<k-1)}
\end{aligned}
$$

Remark 1.4. Theorem 1.3 is a quaternionic analogue of [PS1, Theorem 1.1 and Theorem 5.12]) and generalizes [LV2, §3] to the non-critical slope case.

For the slope zero subspace, we obtain an integrally refined control theorem, which refines $[\mathbf{L V 2}, \S 3]$.

Corollary 1.5 (Corollary 6.8). There exist Hecke-equivariant isomorphisms

$$
\begin{aligned}
S^{N^{-}}\left(N^{+} p, \mathscr{D}_{k}(\mathcal{O})\right)^{(0)} & \stackrel{\simeq}{\longrightarrow} S^{N^{-}}\left(N^{+} p, \mathbf{D}_{k}(\mathcal{O})\right)^{(0)} \\
& \simeq S_{k}^{N^{-}}\left(N^{+} p, \mathcal{O}\right)^{(0)}
\end{aligned}
$$

1.5. Overconvergent construction of $\boldsymbol{p}$-adic $\boldsymbol{L}$-functions. Using the explicit Gross points, we are able to construct anticyclotomic $p$-adic $L$-functions of modular forms of non-critical slope. The following theorem generalizes the constructions of Bertolini-Darmon [BD1], [BD5], Bertolini-Darmon-Iovita-Spiess [BDIS], and Chida-Hsieh $[\mathbf{C H}]$. This also can be regarded as a quaternionic analogue of $[\mathbf{P S 1}, \S 6]$.

Theorem 1.6. Let $f_{k}$ be a newform of slope $h<k-1$ and $\Phi_{f_{k}}$ be the corresponding overconvergent quaternionic form. Then there exists an element $\varsigma^{(1)} \in \widehat{B}^{\times}$(Definition 2.2) such that $\Phi_{f_{k}}\left(\varsigma^{(1)}\right)$ is the h-admissible distribution (Definition 7.4) which defines one half of the anticyclotomic p-adic L-functions of $f_{k}$ (Definition 7.8) and satisfies the expected interpolation property (Corollary 8.9).

1.6. Comparison with the former work. We summarize the comparison with the former work.

- Gross proved the interpolation formula for weight two forms of prime level with the twist by unramified ring class character in [Gro], and the formula is generalized to the weight two forms of arbitrary level and ring class characters of arbitrary conductor and finite order in [Zha, Theorem 7.1]. 
- In [BD1] and [BD5] the anticyclotomic $p$-adic $L$-functions for $p$-ordinary $p$-stabilized newforms of weight two with the twist by ring class characters of $p$-power conductor and of finite order are constructed via a Stickelberger type argument.

- In [BDIS] the construction generalizes to $p$-newforms (the exceptional zero case) of even weight with the twist of unramified ring class characters of finite order. It can be regarded as an overconvergent construction due to [BDIS, (8)] using the $p$-adic integration on $\mathbb{P}^{1}\left(\mathbb{Q}_{p}\right)$ à la Schneider-Teitelbaum. In this construction, a property of $p$-newforms is used essentially. The interpolation formula for higher weight forms is given in [BDIS, Proposition 2.16] only for unramified character twists, and the formula for ring class characters of $p$-power conductor is stated as a conjecture [BDIS, Conjecture 2.17]. Indeed, [CH, Proposition 4.3] proves [BDIS, Conjecture 2.17] as stated in [CH, remark after Proposition 4.3]. See also [Yua].

- In [BD6] the construction generalizes to $p$-ordinary $p$-stabilized newforms but it only allows genus characters [BD6, p. 412] for the character twist. The construction depends heavily on a quaternionic variant of Hida theory and the Hida theory there does not preserve the integrality.

- In $[\mathbf{C H}]$ their construction works for $p$-ordinary $p$-stabilized newforms with limitation of weight $k<p+2$ but with much more general twists by any locally algebraic $p$-adic characters of weight $(i,-i)$ with $-k / 2<i<k / 2$ as described in $[\mathbf{C H}$, Introduction]. Here, the restriction of weight comes from the integrality and $\mu$-invariant issues. Also, Gross points are explicitly constructed at the level of $\widehat{B}^{\times}$. It is very important in our construction.

- In $[\mathbf{C L}]$ and $[\mathbf{C K L}]$ the construction works for $p$-ordinary $p$-stabilized newforms with the twist by same characters as in $[\mathbf{C H}]$. This method uses an integral quaternionic Hida theory and big Gross points.

- In this article the construction works for $p$-stabilized newforms of non-critical slope and allows the twist by any locally algebraic $p$ adic character of weight $(i,-i)$ with $-k / 2<i<k / 2$ arising from an anticyclotomic Hecke character (cf. [CH, §4.2]). If the form is ordinary, then more character twists are allowed as in the case of $[\mathbf{C H}$, Theorem 4.6]. However, the interpolation formula is given only by $p$-power congruences (Corollary 8.9) unless the form is ordinary or of weight two. 
1.7. Organization. In $\S 2$, we review the explicit construction of Gross points following Chida-Hsieh, which is a key input of this work. In $\S 3$, we give a geometric interpretation of the explicit Gross points for the case of weight two forms. In $\S 4$, we compare these two Gross points. We also review other descriptions of Gross points. In $\S 5$, we fix the convention of the coefficient modules for quaternionic forms. In $\S 6$, we review quaternionic forms, introduce their overconvergent variants, and prove the control theorem (Theorem 6.6). In $\S 7$, we give the overconvergent construction of the distribution (Definition 7.4) using the explicit Gross point, which is one half of the $p$-adic $L$-function. Also we recover classical theta elements from the distribution. In $\S 8$, we prove the "weak" interpolation formula (Corollary 8.9) for the distribution using the formula of Chida-Hsieh (Theorem 8.6). In $\S 9$, we give some speculations and ask questions we do not have answers yet.

\section{Explicit Gross points à la Chida and Hsieh}

We very closely follow [CH, $\S 2.1$ and $\S 2.2]$ for the explicit construction. The novelty of this explicit construction of Gross points given by Chida and Hsieh is that the points lie at the level of $\widehat{B}^{\times}$. This allows us to consider the Gross points at the "deepest" level. This explicit approach shows us that it seems more natural to look at the "spaces at certain infinite levels" for the construction of $p$-adic $L$-functions.

Also, in the case of weight two forms, these Gross points can be realized purely geometrically in terms of the Bruhat-Tits tree for $\operatorname{PGL}_{2}\left(\mathbb{Q}_{p}\right)$. We will see this in the next section (§3).

Remark 2.1 (on the tame level structure on the domain). The domain of modular symbols $\operatorname{Div}^{0}\left(\mathbb{P}^{1}(\mathbb{Q})\right)$ is completely independent of level structure and the information of the level structure entirely lies in congruence subgroups. However, the domain of quaternionic forms $B^{\times} \backslash \widehat{B}^{\times} / \widehat{R}^{(p), \times}$ depends on its tame level structure obviously. Thus, the shape of Gross points depends on the tame level structure.

2.1. Explicit setup. Let $K$ be the imaginary quadratic field of discriminant $-D_{K}<0$. Define

$$
\vartheta:= \begin{cases}\frac{D_{K}-\sqrt{-D_{K}}}{2} & \text { if } 2 \nmid D_{K}, \\ \frac{D_{K}-2 \sqrt{-D_{K}}}{4} & \text { if } 2 \mid D_{K},\end{cases}
$$

so that $\mathcal{O}_{K}=\mathbb{Z}+\mathbb{Z} \vartheta$. 
Let $B$ be the definite quaternion algebra over $\mathbb{Q}$ of discriminant $N^{-}$ and level $N^{+}$under Assumption 1.2. Then there exists an embedding of $K$ into $B$ ([Vig, $\S 3$ of chapitre II and $\S 5$.C of chapitre III]). More explicitly, we choose a $K$-basis $(1, J)$ of $B$ so that $B=K \oplus K \cdot J$ such that

(1) $\beta:=J^{2} \in \mathbb{Q}^{\times}$with $\beta<0$,

(2) $J \cdot t=\bar{t} \cdot J$ for all $t \in K$,

(3) $\beta \in\left(\mathbb{Z}_{q}^{\times}\right)^{2}$ for all $q \mid p N^{+}$,

(4) $\beta \in \mathbb{Z}_{q}^{\times}$for all $q \mid D_{K}$.

Fix a square $\operatorname{root} \sqrt{\beta} \in \overline{\mathbb{Q}}$ of $\beta$. Fix an isomorphism

$$
i:=\prod i_{q}: \widehat{B}^{\left(N^{-}\right)} \simeq \mathrm{M}_{2}\left(\mathbb{A}^{\left(N^{-} \infty\right)}\right)
$$

as follows:

(1) For each finite place $q \mid N^{+} p$, the isomorphism

$$
i_{q}: B_{q} \simeq \mathrm{M}_{2}\left(\mathbb{Q}_{q}\right)
$$

by

$$
\begin{aligned}
& i_{q}(\vartheta)=\left(\begin{array}{cc}
\operatorname{trd}(\vartheta) & -\operatorname{nrd}(\vartheta) \\
1 & 0
\end{array}\right), \\
& i_{q}(J)=\sqrt{\beta} \cdot\left(\begin{array}{cc}
-1 & \operatorname{trd}(\vartheta) \\
0 & 1
\end{array}\right),
\end{aligned}
$$

where trd and nrd are the reduced trace and the reduced norm on $B$, respectively. Note that $\sqrt{\beta} \in \mathbb{Z}_{q}^{\times}$here.

(2) For each finite place $q \nmid p N^{+}$, the isomorphism

$$
i_{q}: B_{q} \simeq \mathrm{M}_{2}\left(\mathbb{Q}_{q}\right)
$$

is chosen so that

$$
i_{q}\left(\mathcal{O}_{K} \otimes \mathbb{Z}_{q}\right) \subseteq \mathrm{M}_{2}\left(\mathbb{Z}_{q}\right) .
$$

We fix an embedding $i_{K}: B \hookrightarrow \mathrm{M}_{2}(K)$ defined by $a+b J \mapsto\left(\begin{array}{c}a \\ \frac{b}{b}\end{array} \frac{\bar{a}}{a}\right)$ and define $i_{\mathbb{C}}: B \hookrightarrow \mathrm{M}_{2}(\mathbb{C})$ by $\iota_{\infty} \circ i_{K}$.

2.2. The construction of the points. Fix a decomposition $N^{+} \mathcal{O}_{K}=$ $\mathfrak{N}^{+} \cdot \overline{\mathfrak{N}^{+}}$, which corresponds to the choice of the orientations of local Eichler orders at primes dividing $N^{+}$. We define the local Gross point $\varsigma_{q} \in B_{q}^{\times}$for any rational prime $q$.

2.2.1. $q \nmid N^{+} p$. Let $q \nmid N^{+} p$ be a prime. Then

$$
\varsigma_{q}:=1
$$

in $B_{q}^{\times}$. 
2.2.2. $q \mid N^{+}$. Let $q \mid N^{+}$be a prime, and write $q=\mathfrak{q} \overline{\mathfrak{q}}$ in $\mathcal{O}_{K}$. Then

$$
\varsigma_{q}:=\frac{1}{\sqrt{D_{K}}} \cdot\left(\begin{array}{ll}
\vartheta & \bar{\vartheta} \\
1 & 1
\end{array}\right) \in \mathrm{GL}_{2}\left(K_{\mathfrak{q}}\right)=\mathrm{GL}_{2}\left(\mathbb{Q}_{q}\right)
$$

2.2.3. $q=p$. Suppose that $p=\mathfrak{p} \overline{\mathfrak{p}}$ splits in $K$. Then we put

$$
\varsigma_{p}^{(n)}=\left(\begin{array}{cc}
\vartheta & -1 \\
1 & 0
\end{array}\right)\left(\begin{array}{cc}
p^{n} & 0 \\
0 & 1
\end{array}\right) \in \mathrm{GL}_{2}\left(K_{\mathfrak{p}}\right)=\mathrm{GL}_{2}\left(\mathbb{Q}_{p}\right) .
$$

Suppose that $p$ is inert in $K$. Then we put

$$
\varsigma_{p}^{(n)}=\left(\begin{array}{cc}
0 & 1 \\
-1 & 0
\end{array}\right)\left(\begin{array}{cc}
p^{n} & 0 \\
0 & 1
\end{array}\right) \in \mathrm{GL}_{2}\left(K_{p}\right)=\mathrm{GL}_{2}\left(\mathbb{Q}_{p^{2}}\right) .
$$

\subsubsection{Putting it all together.}

Definition 2.2 (Explicit Gross points). We define the explicit Gross point $\varsigma^{(n)}$ of conductor $p^{n}$ on $\widehat{B}^{\times}$by

$$
\varsigma^{(n)}:=\varsigma_{p}^{(n)} \times \prod_{q \neq p} \varsigma_{q} \in \widehat{B}^{\times} .
$$

2.3. Anticyclotomic Galois action on Gross points. We define the map

$$
x_{n}: \widehat{K}^{\times} \longrightarrow \widehat{B}^{\times}
$$

by

$$
x_{n}(\xi):=\xi \cdot \varsigma^{(n)},
$$

where the action is given by the embedding $K$ into $B$ chosen in $\S 2.1$. Then the set of all points $\left\{x_{n}(\xi): \xi \in \mathbb{A}_{K}^{(\infty), \times}\right\}$ is called twisted explicit Gross points of conductor $p^{n}$.

We also define the map

$$
x_{n}^{-1}: \widehat{K}^{\times} \longrightarrow \widehat{B}^{\times}
$$

by

$$
x_{n}^{-1}(\xi):=\xi^{-1} \cdot \varsigma^{(n)} .
$$

Note that $x_{n}^{-1}(\xi)$ does not appear as $x_{n}(\xi)$ since the inverse map $K^{\times} \rightarrow$ $K^{\times}$by $x \mapsto 1 / x$ does not propagate its domain to $K$.

2.4. Families of optimal embeddings. Let $\mathcal{O}_{n}=\mathbb{Z}+p^{n} \mathcal{O}_{K}$ be the order of $K$ of conductor $p^{n}$. Let $R_{N^{+} p^{r}}$ be an Eichler order of level $N^{+} p^{r}$ prime to $N^{-}$. By the argument in [CH, $\left.§ 2.2\right]$, the embedding of $K$ into $B$ is an optimal embedding of $\mathcal{O}_{n}$ into the Eichler order $B \cap \varsigma^{(n)} \cdot \widehat{R}_{N^{+} p^{r}}^{\times}$. $\left(\varsigma^{(n)}\right)^{-1}$ if $r \leq n$, i.e.

$$
\left(B \cap \varsigma^{(n)} \cdot \widehat{R}_{N^{+} p^{r}}^{\times} \cdot\left(\varsigma^{(n)}\right)^{-1}\right) \cap K=\mathcal{O}_{n} .
$$

This is used in the comparison among Gross points defined on different domains $(\S 4.2)$. 
Remark 2.3. See $[\mathbf{L V 1}, \S 4.1(12)]$ for another recipe of the families of optimal embeddings. Their recipe calculates the $p$-part only, but the oriented optimal embeddings are determined locally ([LV1, Lemma 4.1]).

\section{Geometric Gross points for weight two forms}

In this section we give a geometric interpretation of the projection of the explicit Gross points to the double coset space at the $\Gamma_{0}\left(p^{\infty}\right)$-level, i.e. the Bruhat-Tits tree. Although it does not give us the full information of the explicit Gross points, it seems to be helpful for a more theoretical understanding of the Gross points. The geometric description naturally shows us a $p$-adic intuition in the construction of anticyclotomic $p$-adic $L$-functions of modular forms (of weight two, at least).

In the construction of the anticyclotomic $p$-adic $L$-functions of an ordinary newform of weight two, we choose an infinite sequence of consecutive vertices $v_{0}, v_{1}, \ldots, v_{n}, \ldots$ without backtracking on $B T_{p}$ (We call them Gross points of conductor $p^{n}$ at level 0 ). The oriented edge $e_{n}$ on $B T_{p}$ whose source is $v_{n}$ and target is $v_{n+1}$ is called Gross points of conductor $p^{n}$ at level 1 . By construction, the sequence of the edges $e_{0}, e_{1}, \ldots, e_{n}, \ldots$ has coherent direction. We also call these two points classical Gross points.

Remark 3.1. Indeed, the first choice $v_{0}$ is an infinite line if $p$ splits in $K$, but we will call it a "vertex" for convenience. See [DI, Figure 1] for the picture.

The goal of this section is to reinterpret these infinite choices of classical Gross points at the $\Gamma_{0}\left(p^{r}\right)$ and $\Gamma_{0}\left(p^{\infty}\right)$-levels. The "Gross points at the $\Gamma_{0}\left(p^{\infty}\right)$-level" will be called geometric Gross points (Definition 3.11).

3.1. Galois-theoretic setup. Let $\mathcal{O}_{K}$ be the ring of integers of $K$ and $\mathcal{O}_{K}\left[\frac{1}{p}\right]$ be the maximal $\mathbb{Z}\left[\frac{1}{p}\right]$-order in $K$. Let

$$
\widetilde{G}_{\infty}=K^{\times} \backslash \widehat{K}^{\times} /\left(\widehat{\mathbb{Q}}^{\times} \cdot \prod_{\ell \neq p} \mathcal{O}_{K}\left[\frac{1}{p}\right]_{\ell}^{\times}\right)
$$

be the Galois group of the ring class field $\widetilde{K}_{\infty}$ of $K$ of conductor $p^{\infty}$ so that $\widetilde{G}_{\infty}=\operatorname{Gal}\left(\widetilde{K}_{\infty} / K\right)$.

Choice 3.2. We choose an oriented optimal embedding $\Psi_{0}: K \rightarrow B$ such that $\Psi_{0}(K) \cap R[1 / p]=\Psi_{0}\left(\mathcal{O}_{K}[1 / p]\right)$ which is as equivalent as the choice in $§ 2.1$.

With Choice $3.2 \Psi_{0}$ induces a family of optimal embeddings $\Psi_{p^{r}}$ such that $\Psi_{p^{r}}(K) \cap R_{N^{+} p^{r}}=\Psi_{p^{r}}\left(\mathcal{O}_{r}\right)$ for all $r \geq 0$ as in $\S 2.4$. 
3.2. Classical Gross points: Gross points at level 0 and 1. Let $B T_{p}$ be the Bruhat-Tits tree for $\mathrm{PGL}_{2}\left(\mathbb{Q}_{p}\right)$. The action of $\mathrm{PGL}_{2}\left(\mathbb{Q}_{p}\right)$ on $B T_{p}$ is given via right conjugation. The chosen embedding $\Psi_{0}$ induces the $p$-adic embedding $\left(\Psi_{0}\right)_{p}: K_{p}^{\times} / \mathbb{Q}_{p}^{\times} \hookrightarrow B_{p}^{\times} / \mathbb{Q}_{p}^{\times} \simeq \operatorname{PGL}_{2}\left(\mathbb{Q}_{p}\right)$. This embedding yields the action of $K_{p}^{\times} / \mathbb{Q}_{p}^{\times}$on $B T_{p}$ via left translation. The structure of $\widetilde{G}_{\infty}$ can be described by the following short exact sequence:

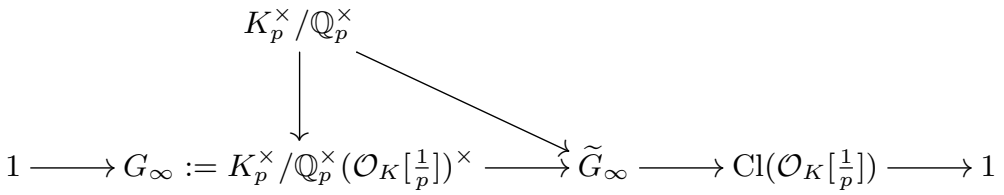

Remark 3.3. The class group $\mathrm{Cl}\left(\mathcal{O}_{K}\left[\frac{1}{p}\right]\right)$ permutes oriented optimal embeddings transitively, and the permutation is explicitly defined in [BDIS, $\S 2.3]$.

Consider the decreasing filtration of $K_{p}^{\times} / \mathbb{Q}_{p}^{\times}$

$$
\cdots \subseteq U_{n+1} \subseteq U_{n} \subseteq U_{n-1} \subseteq \cdots \subseteq U_{1} \subseteq U_{0} \subseteq K_{p}^{\times} / \mathbb{Q}_{p}^{\times},
$$

where $U_{0}$ is the maximal compact subgroup of $K_{p}^{\times} / \mathbb{Q}_{p}^{\times}$and $U_{n}=(1+$ $\left.p^{n} \mathcal{O}_{K} \otimes_{\mathbb{Z}} \mathbb{Z}_{p}\right) /\left(1+p^{n} \mathbb{Z}_{p}\right)$ for each $n \geq 1$. Let $G_{n}:=G_{\infty} /($ the image of $U_{n}$ in $\left.G_{\infty}\right)$ and $\widetilde{G}_{n}:=\widetilde{G}_{\infty} /\left(\right.$ the image of $U_{n}$ in $\left.\widetilde{G}_{\infty}\right)$.

Choice 3.4. We choose a sequence of consecutive vertices $v_{0}, v_{1}, v_{2}, \ldots$ of $V\left(B T_{p}\right)$ with the coherent orientation and without backtracking such that $\operatorname{Stab}_{\left(\Psi_{0}\right)_{p}\left(K_{p}^{\times} / \mathbb{Q}_{p}^{\times}\right)}\left(v_{n}\right)=U_{n}$ for all $n \geq 0$ as equivalent as the choice in §2.2.3 and §2.2.4.

Definition 3.5 (Classical Gross points).

(1) Each vertex $v_{n}$ in the chosen sequence is called a (classical) Gross point of conductor $p^{n}$ at level 0 .

(2) Each oriented edge $e_{n}=\left(v_{n} \rightarrow v_{n+1}\right)$ is called a (classical) Gross point of conductor $p^{n}$ at level 1 .

Remark 3.6 (on the domain of Gross points). Often in the literature, classical Gross points are defined on the quotient graphs of $B T_{p}$, which are equivalent to the double coset spaces via strong approximation. However, both Gross points on the tree and on the quotient graph give exactly the same result since quaternionic forms are invariant under the quotient. It seems difficult to observe Gross points at higher level on the quotient graph intuitively since the images of length $n$ line segments in the quotient graph may have very random shapes due to the complication of the quotient graph. For the computation of the graph, see $[\mathbf{F M}]$. 
Recently, it seems that this complication has an application to cryptography, so called isogeny based cryptography. For example, see [DJP, $\S 2.2]$.

3.3. A simple observation: towards higher and infinite level. A natural idea toward the Gross points on a certain space at the infinite level begins with the following question.

Question 3.7. How can we regard a coherent infinite sequence of classical Gross points itself as one element in a more suitable domain than the set of vertices or oriented edges of the Bruhat-Tits tree?

We recall a strong approximation result.

Proposition 3.8 ([BD5, $\S 1.2(16)])$. The embedding into the p-th place

$$
\begin{aligned}
\left(R\left[\frac{1}{p}\right]\right)^{\times} \backslash B_{p}^{\times} / \mathbb{Q}_{p}^{\times} & \simeq B^{\times} \backslash \widehat{B}^{\times} /\left(\widehat{\mathbb{Q}}^{\times} \prod_{\ell \neq p} R_{\ell}^{\times}\right) \\
b_{p} & \longmapsto\left(1, \ldots, 1, b_{p}, 1, \ldots\right)
\end{aligned}
$$

is a canonical bijection.

Let $\vec{E}_{r}\left(B T_{p}\right)$ be the set of consecutive line segments of length $r$ with coherent orientation of $B T_{p}$ without backtracking. Let $v_{0}, v_{1}, v_{2}, \ldots, v_{r}$ be the sequence of consecutive vertices of $B T_{p}$ whose stabilizers are $\mathbb{Q}_{p}^{\times}$. $\mathrm{GL}_{2}\left(\mathbb{Z}_{p}\right), \mathbb{Q}_{p}^{\times} \cdot \gamma^{-1} \mathrm{GL}_{2}\left(\mathbb{Z}_{p}\right) \gamma, \ldots, \mathbb{Q}_{p}^{\times} \cdot \gamma^{-r} \mathrm{GL}_{2}\left(\mathbb{Z}_{p}\right) \gamma^{r}$ with $\gamma=\left(\begin{array}{cc}p & 0 \\ 0 & 1\end{array}\right)$, respectively. Thus, the whole sequence is an element of $\vec{E}_{r}\left(B T_{p}\right)$. Then the stabilizer of the whole sequence is $\mathbb{Q}_{p}^{\times} \cdot R_{r, p}^{\times}$. We observe the following statement.

Proposition 3.9. The $\mathrm{GL}_{2}\left(\mathbb{Q}_{p}\right)$-orbit of the sequence of consecutive vertices $v_{0}, v_{1}, v_{2}, \ldots, v_{r}$ without backtracking is $\vec{E}_{r}\left(B T_{p}\right)$.

Proof: Since the action of $\mathrm{GL}_{2}\left(\mathbb{Q}_{p}\right)$ on $B T_{p}$ preserves distance, the $\mathrm{GL}_{2}\left(\mathbb{Q}_{p}\right)$-orbit of the sequence $v_{0}, v_{1}, v_{2}, \ldots, v_{r}$ is a subset of $\vec{E}_{r}\left(B T_{p}\right)$. It suffices to show the action of $\mathrm{GL}_{2}\left(\mathbb{Q}_{p}\right)$ on $\vec{E}_{r}\left(B T_{p}\right)$ is transitive. Let $w_{0}, w_{1}, w_{2}, \ldots, w_{r-1}, w_{r}$ be an arbitrary element of $\vec{E}_{r}\left(B T_{p}\right)$. Since $\mathrm{GL}_{2}\left(\mathbb{Q}_{p}\right)$ acts transitively on $\vec{E}_{1}\left(B T_{p}\right)$, we may assume $w_{r-1}=v_{r-1}$ and $w_{r}=v_{r}$. Now we apply induction on $r$ in the decreasing direction. Let $k<r$ be the smallest integer such that $w_{k}=v_{k}, w_{k+1}=v_{k+1}, \ldots$, and $w_{r}=v_{r}$. The stabilizer of the sequence $w_{k}, \ldots w_{r}$ consists of the matrices $\left(\begin{array}{cc}a & b / p^{k} \\ p^{r} c & d\end{array}\right) \in \mathrm{GL}_{2}\left(\mathbb{Q}_{p}\right)$ with $a, b, c, d \in \mathbb{Z}_{p}$.

Then $w_{k-1}$ corresponds to the homothety class of the lattice

$$
\left(\mathbb{Z}_{p} \times \mathbb{Z}_{p}\right) \cdot\left(\begin{array}{cc}
p^{k} & \Delta \\
0 & p
\end{array}\right)
$$


for some $\Delta=1, \ldots p-1$. Multiplying $\left(\begin{array}{c}1-\Delta / p^{k} \\ 0 \\ 1\end{array}\right)$ on the right, the lattice corresponding to $w_{k-1}$ changes to the lattice $\left(\mathbb{Z}_{p} \times \mathbb{Z}_{p}\right) \cdot\left(\begin{array}{cc}p^{k-1} & 0 \\ 0 & 1\end{array}\right)$ upto homothety. Since the corresponding vertex is $v_{k-1}$ and $\left(\begin{array}{c}1-\Delta / p^{k} \\ 0\end{array}\right)$ is in the stabilizer of the sequence $v_{k}, \ldots, v_{r}$, we reduce $k$ to $k-1$ by multiplying $\left(\begin{array}{cc}1 & -\Delta / p^{k} \\ 0 & 1\end{array}\right)$ on the right. Repeating the process, we obtain the conclusion.

Then Proposition 3.9 and the orbit-stabilizer theorem show that there exist bijections:

$$
\vec{E}_{r}\left(B T_{p}\right) \stackrel{\text { Prop. 3.9 }}{\simeq}\left(v_{0}, \ldots, v_{r}\right) \cdot \mathrm{GL}_{2}\left(\mathbb{Q}_{p}\right) \underset{\text { orbit-stabilizer }}{\simeq} \mathrm{GL}_{2}\left(\mathbb{Q}_{p}\right) /\left(\mathbb{Q}_{p}^{\times} \cdot R_{r, p}^{\times}\right) .
$$

This identification gives us a hint to define the case of $r=\infty$.

We define

$$
\vec{E}_{\infty}\left(B T_{p}\right):=\mathrm{GL}_{2}\left(\mathbb{Q}_{p}\right) /\left(\mathbb{Q}_{p}^{\times} \cdot R_{N^{+} p^{\infty}, p}^{\times}\right) \simeq\left(v_{0}, v_{1}, \ldots\right) \cdot \mathrm{GL}_{2}\left(\mathbb{Q}_{p}\right) .
$$

Then each element here has interpretation as an infinite consecutive sequence of vertices from a vertex to a boundary of the Bruhat-Tits tree since each element has the form $\left(v_{0}, v_{1}, \ldots\right) \cdot \gamma$ where $\gamma \in \mathrm{GL}_{2}\left(\mathbb{Q}_{p}\right)$. Also, $\vec{E}_{\infty}\left(B T_{p}\right)$ admits natural quotient maps

$$
\begin{aligned}
\vec{E}_{\infty}\left(B T_{p}\right) & \longrightarrow \vec{E}_{r}\left(B T_{p}\right) \\
\left(v_{0}, v_{1}, \ldots\right) & \longmapsto\left(v_{0}, v_{1}, \ldots, v_{r}\right)
\end{aligned}
$$

for all $r \geq 0$. Note that the stabilizer of $v_{0}$ is $U_{0}$ and the stabilizer of a boundary of $B T_{p}$, an element in $\mathbb{P}^{1}\left(\mathbb{Q}_{p}\right)$, is trivial.

We are now able to give a heuristic definition of geometric Gross points and a more group-theoretic and axiomatic definition is given in $\S 3.4$.

Definition 3.10 (Heuristic definition of geometric Gross points). A geometric Gross point is the consecutive sequence of classical Gross points at level 0 depending on Choice 3.2, Choice 3.4, and Definition 3.5 in $\vec{E}_{\infty}\left(B T_{p}\right)$.

3.4. A group theoretic realization and independence. We give a more axiomatic definition of geometric Gross points. For notational convenience, let

- $G=\mathrm{GL}_{2}\left(\mathbb{Q}_{p}\right)$,

- $B=$ the upper Borel subgroup of $G$,

- $K_{0}=\mathrm{GL}_{2}\left(\mathbb{Z}_{p}\right)$,

- $K_{r}=\Gamma\left(p^{r} \mathbb{Z}_{p}\right)$, and

- $Z=$ the center of $G \simeq \mathbb{Q}_{p}^{\times}$. 
Then the Iwasawa decomposition implies that $G=B \cdot K$. We have natural projection maps

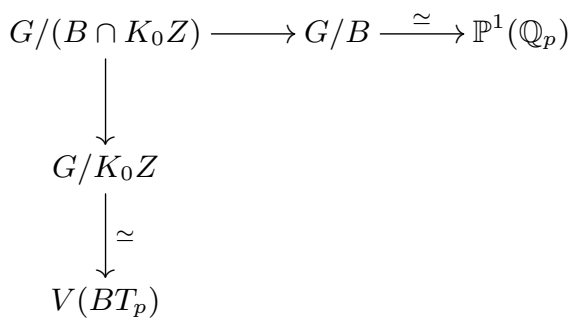

and embedding

$$
G /\left(B \cap K_{0} Z\right) \longleftrightarrow V\left(B T_{p}\right) \times \mathbb{P}^{1}\left(\mathbb{Q}_{p}\right) .
$$

We remark that we do not know how to characterize the image explicitly.

We axiomatize Definition 3.10.

Definition 3.11 (Geometric Gross points). Fix a central Gross point/ line $v_{0}$ of conductor 1 and level 0 . For $n \geq 0$, an element $\sigma^{(n)} \in G /(B \cap$ $\left.K_{0} Z\right)$ is a geometric Gross point of conductor $p^{n}$ if

(1) the image of $\sigma^{(0)}$ in $G / K_{0} Z$ is $v_{0}$ under the natural projection,

(2) the image of $\sigma^{(n)}:=\sigma^{(0)} \cdot\left(\begin{array}{cc}p^{n} & 0 \\ 0 & 1\end{array}\right)$ in $G / K_{0} Z$ has stabilizer $U_{n}$ under the action of $K_{p}^{\times} / \mathbb{Q}_{p}^{\times}$via the chosen optimal embedding $\Psi_{0}$, and

(3) the image of $\sigma^{(n)}$ in $G / B$ does not change for all $n \geq 0$.

Proposition 3.12 (Uniqueness and Galois properties).

(1) By the embedding, Properties (1) and (3) in Definition 3.11 uniquely determine a point in $G /\left(B \cap K_{0} Z\right)$.

(2) The image of $\sigma^{(r)}$ in $G /\left(Z \cdot R_{N^{+} p^{r}, p}^{\times}\right)$is $\left(v_{0}, \ldots, v_{r}\right)$ where $v_{i}=$ $v_{i-1} \cdot\left(\begin{array}{cc}p & 0 \\ 0 & 1\end{array}\right)$.

Proof: (1) Obvious since $G /\left(B \cap K_{0} Z\right) \hookrightarrow V\left(B T_{p}\right) \times \mathbb{P}^{1}\left(\mathbb{Q}_{p}\right)$.

(2) The image of $\sigma^{(0)}$ under the natural quotient map $G /\left(B \cap K_{0} Z\right) \rightarrow$ $G / K_{0} Z$ is $v_{0}$ and a lifing $v_{0}$ to $G /\left(Z \cdot R_{N^{+} p^{r}, p}^{\times}\right)$gives a length $r$ line segment whose target endpoint is $v_{0}$. Shifting by $\left(\begin{array}{cc}p^{r} & 0 \\ 0 & 1\end{array}\right)$ gives the conclusion.

For a given optimal embedding $\Psi_{0}: K^{\times} \rightarrow B^{\times}$, we define the reversed embedding $\Psi_{0}^{-1}: K^{\times} \rightarrow B^{\times}$by $a \mapsto \Psi_{0}\left(a^{-1}\right)$. Then, for $n \geq 0$, we define the dual geometric Gross point to $\sigma^{(n)}$ by an element $\sigma^{(n), *} \in$ $G /\left(B \cap K_{0} Z\right)$ as exactly same as Definition 3.11 but with the reversed embedding $\Psi_{0}^{-1}$ in Condition (2). 
Proposition 3.13 (Independence of choices). Let $\sigma_{1}^{(0)}, \sigma_{2}^{(0)}$ be two geometric Gross points. Then they differ only by the translation by an element of $K_{p}^{\times} / \mathbb{Q}_{p}^{\times}$.

Proof: We split the proof into two parts depending on whether $p$ is inert in $K$ or splits in $K$. This is a slightly refined version of [BD4, Lemma 4.3].

The inert case: From [BD2, Lemma 2.7], we can deduce $\widetilde{G}_{n}$ acts transitively on the classical Gross points of conductor $p^{n}$ for any $n$. With [BD2, Lemma 2.8], it is easy to see that $\widetilde{G}_{n}$ acts transitively on higher Gross points of conductor $p^{n}$. Note that the subquotient $K_{p}^{\times} / \mathbb{Q}_{p}^{\times}$of $\widetilde{G}_{\infty}$ acts on $\mathbb{P}^{1}\left(\mathbb{Q}_{p}\right)$ simply transitively due to $[\mathbf{B D} 4, \S 4.1$, Step 2]. This ensures that $\widetilde{G}_{\infty}$ acts on the set of Gross points at infinite level transitively.

The split case: We can deduce the same conclusion for higher Gross points of conductor $p^{n}$ following the argument in [BD3, §3]. However, $K_{p}^{\times} / \mathbb{Q}_{p}^{\times}$does not act on $\mathbb{P}^{1}\left(\mathbb{Q}_{p}\right)$ transitively in this case. It has 3 orbits: $0, \infty$, and $\mathbb{Q}_{p}^{\times}$. See $[$BD3, $\S 7 . \mathrm{I}]$ for detail. However, any sequence of classical Gross points does not converges to 0 or $\infty$ in $\mathbb{P}^{1}\left(\mathbb{Q}_{p}\right)$ in this case. See [DI, §2.2 and Figure 1] for detail.

\section{Comparison among Gross points}

4.1. Comparison of explicit and geometric Gross points. Considering the strong approximation for quaternion algebras (Proposition 3.8), we observe more precise relations among the double coset spaces as follows:

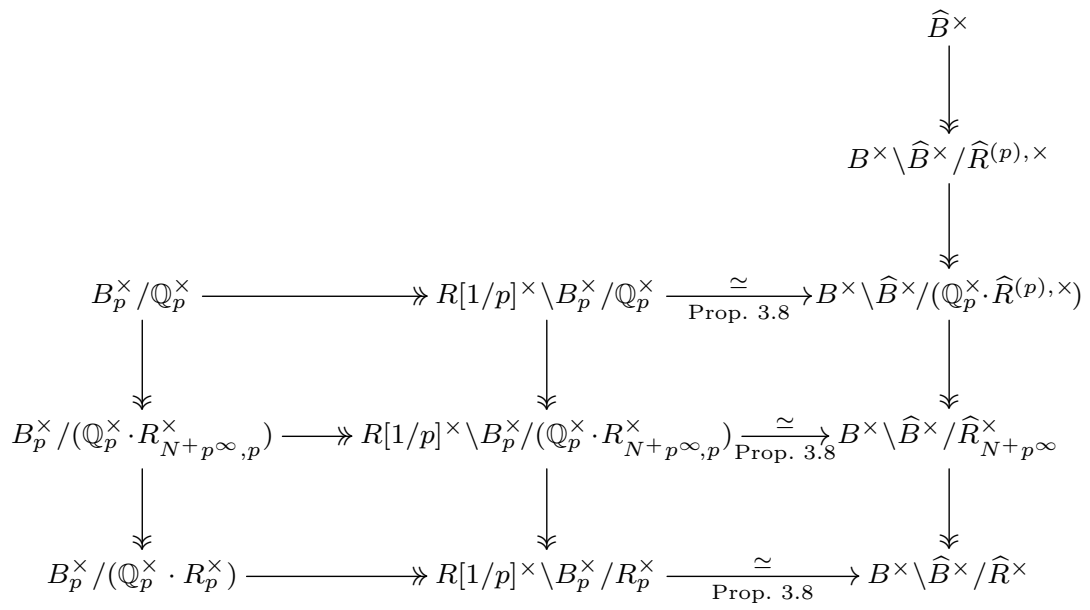


There is no level structure at $p$ in $B^{\times} \backslash \widehat{B}^{\times} / \widehat{R}^{(p), \times}, B^{\times} \backslash \widehat{B}^{\times} / \widehat{R}_{N^{+} p^{\infty}}^{\times}$has $\Gamma_{0}\left(p^{\infty}\right)$-level structure, and $B^{\times} \backslash \widehat{B}^{\times} / \widehat{R}^{\times}$has full level structure at $p$. Note that $B^{\times} \backslash \widehat{B}^{\times} / \widehat{R}^{(p), \times}$ is the domain for quaternionic forms of arbitrary weight and $B^{\times} \backslash \widehat{B}^{\times} / \widehat{R}^{\times}$is the domain for quaternionic forms of weight two only.

Let

- $\varsigma^{(n)}$ be the explicit Gross point on $\widehat{B}^{\times}$defined in Definition 2.2,

- $\sigma^{(n)}$ be the geometric Gross point on $B_{p}^{\times} /\left(\mathbb{Q}_{p}^{\times} \cdot R_{N^{+} p^{\infty}, p}^{\times}\right)$defined in Definition 3.11, and

- $v_{n}$ be the classical Gross point on $B_{p}^{\times} /\left(\mathbb{Q}_{p}^{\times} \cdot R_{p}^{\times}\right)$defined in Definition 3.5.

The classical Gross points $v_{n}$ and the geometric Gross points coincide $\sigma^{(n)}$ by Proposition 3.12(2). The classical Gross points $v_{n}$ and the explicit Gross points $\varsigma^{(n)}$ coincide by the construction of the explicit Gross points and theta elements in [CH, §4.1] (cf. Choice 3.2). Thus, these points coincide in the above diagram as follows:

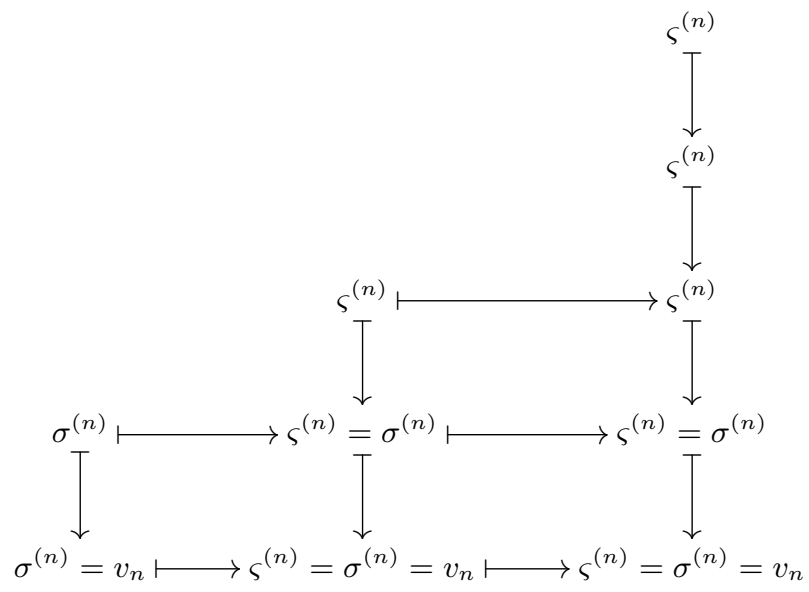

4.2. Comparison with other Gross points. We also consider other definitions of Gross points and the relation with them. All the Gross points here correspond to the classical one (of level 0 ).

Definition 4.1 (Other definitions of Gross points).

(1) Consider the $K$-points of the Gross curve of level $N^{+}$and discriminant $N^{-}$

$$
B^{\times} \backslash \widehat{B}^{\times} \times \operatorname{Hom}(K, B) / \widehat{R}_{N^{+}}^{\times} .
$$

Following [BD1, §2.1], [Lon, §3.1], $\left(x_{n}, \Psi\right)$ is a Gross point of conductor $p^{n}$ on the Gross curve if $\Psi(K) \cap x_{n} \widehat{R}_{N^{+}} x_{n}^{-1}=\Psi\left(\mathcal{O}_{n}\right)$. 
(2) Following [CV, §5.3], [Lon, §4.2], we define the set of Gross points by

$$
\Psi_{0}\left(K^{\times}\right) \backslash \widehat{B}^{\times} / \widehat{R}_{N^{+}}^{\times}
$$

and a Gross point $x_{n} \in \Psi_{0}\left(K^{\times}\right) \backslash \widehat{B}^{\times} / \widehat{R}_{N^{+}}^{\times}$has conductor $p^{n}$ if $\Psi_{0}(K) \cap x_{n} \widehat{R}_{N^{+}} x_{n}^{-1}=\Psi_{0}\left(\mathcal{O}_{n}\right)$.

(3) The equivalence of the above descriptions comes from the map

$$
\Psi_{0}\left(K^{\times}\right) \backslash \widehat{B}^{\times} / \widehat{R}_{N^{+}}^{\times} \longrightarrow B^{\times} \backslash \widehat{B}^{\times} \times \operatorname{Hom}(K, B) / \widehat{R}_{N^{+}}^{\times}
$$

defined by $x_{n} \mapsto\left(x_{n}, \Psi_{0}\right)$. See [Lon, §3.1] for proof.

Remark 4.2. Since we start with a chosen oriented optimal embedding $\Psi_{0}$, all the "CM points" in the original reference become Gross points.

Then it is not difficult to check these Gross points coincide with the classical points (at least at the level of the values of quaternionic forms) by comparing two equivalent construction of theta elements of modular forms of weight two ([BD5, §1.2] with classical Gross points and [BD1, $\S 2.7]$ with Gross points on Gross curves). Their relation can be summarized in the following diagram:

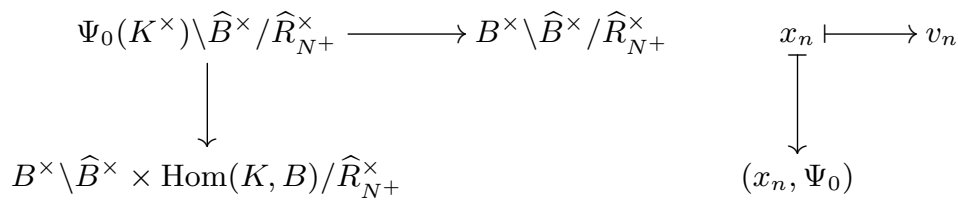

\section{Coefficients}

Let $E$ be a finite extension of $\mathbb{Q}_{p}$ and $\mathcal{O}=\mathcal{O}_{E}$. Let

$$
\Sigma_{0}(p):=\left\{\left(\begin{array}{ll}
a & b \\
c & d
\end{array}\right) \in \mathrm{M}_{2}\left(\mathbb{Z}_{p}\right): c \in p \mathbb{Z}_{p}, d \in \mathbb{Z}_{p}^{\times}, \text {and } a d-b c \neq 0\right\}
$$

be the semigroup we concern to see $U_{p}$-action. It is not as the same one as given in [PS1, §3.3]. More precisely, if $r=\left(\begin{array}{ll}a & b \\ c & d\end{array}\right) \in \Sigma_{0}(p)$ then its adjugate $r^{*}=\left(\begin{array}{cc}d & -b \\ -c & a\end{array}\right)$ satisfies the condition given in [PS1, §3.3]. Since we will define our left action by the adjugate right action given in [PS1, $\S 3.3]$, the specialization maps will be compatible with the convention of [PS1].

5.1. Symmetric powers. Our convention is similar to but not exactly the same as those of $[\mathbf{C H}]$ and $[\mathbf{P S 1}]$. We also introduce an equivariant pairing to obtain the distribution relation later. 
5.1.1. Semigroup action. Let $L_{k}(E):=\operatorname{Sym}^{k-2}\left(E^{2}\right)$ and $L_{k}(\mathcal{O}):=$ $\operatorname{Sym}^{k-2}\left(\mathcal{O}^{2}\right)$. They admit the left actions of $\Sigma_{0}(p)$ and $\mathrm{GL}_{2}\left(\mathbb{Z}_{p}\right)$ via the representation

$$
\rho_{k}: \Sigma_{0}(p)\left(\text { or } \mathrm{GL}_{2}\left(\mathbb{Z}_{p}\right)\right) \subseteq \mathrm{M}_{2}(\mathcal{O}) \longrightarrow \operatorname{End}_{\mathcal{O}}\left(L_{k}(\mathcal{O})\right)
$$

defined by

$$
\left(\rho_{k}(r) \circ P\right)(X, Y)=P\left(r^{*}(X, Y)\right)=P(d X-b Y,-c X+a Y),
$$

where $r=\left(\begin{array}{ll}a & b \\ c & d\end{array}\right) \in \Sigma_{0}(p)$ and $P(X, Y) \in L_{k}(\mathcal{O})$ is a homogeneous polynomial of variables $X, Y$ of degree $k-2$.

5.1.2. An (ad hoc) equivariant pairing. Consider the following perfect $\mathrm{GL}_{2}\left(\mathbb{Q}_{p}\right)$-equivariant pairing:

$$
\begin{aligned}
\langle-,-\rangle_{k}: L_{k} \times L_{k}\left(\operatorname{det}^{2-k}\right) & \longrightarrow E \\
\left(X^{i} \cdot Y^{k-2-i}, X^{k-2-j} \cdot Y^{j}\right) & \longmapsto(-1)^{i} \cdot\left(\begin{array}{c}
k-2 \\
i
\end{array}\right)^{-1} \cdot \delta_{i, j},
\end{aligned}
$$

where $\delta_{i, j}$ is the Kronecker delta. The equivariant property is given as follows:

$$
\left\langle\rho_{k}(r) \circ P_{1}(X, Y), \rho_{k}^{*}(r) \circ P_{2}(X, Y)\right\rangle_{k}=\left\langle P_{1}(X, Y), P_{2}(X, Y)\right\rangle_{k},
$$

where $\rho_{k}^{*}:=\rho_{k} \otimes \operatorname{det}^{2-k}$. We also write $L_{k}(2-k)=L_{k}\left(\operatorname{det}^{2-k}\right)$.

Remark 5.1 (Normalization of $\mathrm{GL}_{2}\left(\mathbb{Q}_{p}\right)$-action). In $[\mathbf{C H}, \S 2.3]$, the action of $\mathrm{GL}_{2}\left(\mathbb{Q}_{p}\right)$ is unitarily normalized, i.e. the action on the both side is given by $\rho_{k} \otimes \operatorname{det}^{\frac{2-k}{2}}$. However, the unitary normalization is not compatible with the integral theory of quaternionic forms. See $\S 6.3$ and Corollary 6.8 .

The pairing itself is not expected to be $p$-integral unless $k-2<p$ since it involves $(k-2)$ ! in the denominator. In other words, we have

$$
\langle-,-\rangle_{k}: L_{k}(\mathcal{O}) \times L_{k}\left({ }^{2-k}\right)(\mathcal{O}) \longrightarrow \frac{1}{(k-2) !} \mathcal{O} .
$$

5.2. Distributions (as coefficient modules). In order to introduce the $p$-adic deformation of quaternionic forms, we record the standard notion of $p$-adic distributions and fix convention here. Since distribution modules themselves are independent of types of $\mathbb{Z}_{p}$-extensions, the argument of [PS1, §3.3 and §3.4] applies to our setting directly. See [PS1, $\S 3]$ for detail.

Let $f(z)$ be a rigid analytic or locally analytic function on $\mathbb{Z}_{p}$. It admits right weight $k$ action of $\Sigma_{0}(p)$ as follows:

$$
\left(\left.f\right|_{k} r\right)(z):=(c z+d)^{k-2} \cdot f\left(\frac{a z+b}{c z+d}\right) .
$$


Let $\mathbf{D}_{k} / \mathscr{D}_{k}$ be the module of $E$-valued rigid analytic/locally analytic distributions on $\mathbb{Z}_{p}$ with left weight $k$ action of $\Sigma_{0}(p)$, respectively. The action is defined as dual:

$$
\left(r \circ \mu_{k}\right)(f(z)):=\mu_{k}\left(\left(\left.f\right|_{k} r\right)(z)\right),
$$

where $\mu_{k} \in \mathbf{D}_{k}$ or $\mathscr{D}_{k}$. Note that there is a natural inclusion $\mathscr{D}_{k} \hookrightarrow \mathbf{D}_{k}$ as in $[\mathbf{P S 1}, \S 3.1]$. We regard the action as the representation $\widetilde{\rho}_{k}: \Sigma_{0}(p) \rightarrow$ $\operatorname{Aut}_{E}\left(D_{k}\right)$ where $D_{k}=\mathbf{D}_{k}$ or $\mathscr{D}_{k}$.

The $\Sigma_{0}(p)$-equivariant specialization map $\operatorname{sp}_{k}: D_{k} \rightarrow L_{k}$ is defined by

$$
\mathrm{sp}_{k}: \mu_{k} \longmapsto \int_{\mathbb{Z}_{p}}(Y-z X)^{k-2} d \mu_{k}(z),
$$

where $D_{k}=\mathbf{D}_{k}$ or $\mathscr{D}_{k}$. We follow $[\mathbf{P S 1}, \S 3.4]$ for the convention of the specialization. Also, we defined the actions of $\Sigma_{0}(p)$ on both sides to make the map equivariant.

Notation 5.2. We omit $\rho_{k}$ and $\widetilde{\rho}_{k}$ if there is no confusion.

\section{Overconvergent quaternionic forms and control theorems}

In this section we define quaternionic forms and their overconvergent variants. We prove the control theorem to compare them. We also give a completed cohomological description of quaternionic forms.

\subsection{Classical $p$-adic quaternionic forms.}

Definition 6.1 (Classical $p$-adic quaternionic forms).

(1) A continuous function $\phi_{k}: B^{\times} \backslash \widehat{B}^{\times} / \widehat{R}^{(p), \times} \rightarrow L_{k}(E)$ is called a p-adic quaternionic form of discriminant $N^{-}$, level $N^{+} p^{r}$, and weight $k$ if $\phi_{k}$ satisfies the following transformation property:

$$
\phi_{k}(\alpha b u)=\rho_{k}\left(u_{p}^{-1}\right) \circ \phi_{k}(b),
$$

where $\alpha \in B^{\times}$and $u \in \widehat{R}_{N^{+} p^{r}}^{\times}$, and $u_{p}$ is the $p$-part of $u$.

(2) The space of such $p$-adic quaternionic forms is denoted by $S_{k}^{N^{-}}\left(N^{+} p^{r}, E\right)$ if $k \neq 2$. If $k=2$, then $S_{k}^{N^{-}}\left(N^{+} p^{r}, E\right)$ denotes the space of $p$-adic quaternionic forms which are not constant.

(3) If one changes the level structure by $U_{r}, Z_{r}$, or other level structures, one may easily define $S_{k}^{N^{-}}\left(N^{+}, p^{r}, E\right), S_{k}^{N^{-}}\left(Z_{r}, E\right)$, or spaces of quaternionic forms of various levels.

Remark 6.2. Our quaternionic forms corresponds to " $\ell$-adic modular forms" or " $\ell$-adic avatar" (with $\ell=p$ ) in $[\mathbf{C H}, \S 4.1]$. See $\S 8.1$ for detail. 
Let $\mathbb{T}_{k}^{N^{-}}\left(N^{+} p^{r}\right)_{E}$ be the full Hecke algebra over $E$ acting faithfully on $S_{k}^{N^{-}}\left(N^{+} p^{r}, E\right)$ and $\mathbb{T}_{k}^{N^{-}}\left(N^{+}, p^{r}\right)_{E}$ be the full Hecke algebra over $E$ acting faithfully on $S_{k}^{N^{-}}\left(N^{+}, p^{r}, E\right)$. We compare their structures with classical modular forms. Let $S_{k}\left(N p^{r}, E\right)^{N^{-} \text {new }}$ or $S_{k}\left(N, p^{r}, E\right)^{N^{-} \text {-new }}$ be the $N^{-}$-new subspace of cuspforms of weight $k$ and level $\Gamma_{0}\left(N p^{r}\right)$ or $\Gamma_{0}(N) \cap \Gamma_{1}\left(p^{r}\right)$ whose Fourier coefficients lie in $E$ and $\mathbb{T}_{k}\left(N^{+} p^{r}\right)_{E}^{N^{-} \text {-new }}$ or $\mathbb{T}_{k}\left(N^{+}, p^{r}\right)_{E}^{N^{-} \text {new }}$ be the corresponding quotient Hecke algebra, respectively.

Then the Jacquet-Langlands correspondence (over fields) shows the following relation between classical modular forms and quaternionic forms.

Theorem 6.3 ([LV2, §3.3]). There exist isomorphisms of Hecke algebras over $E$

$$
\begin{aligned}
\mathbb{T}_{k}^{N^{-}}\left(N^{+} p^{r}\right)_{E} & \simeq \mathbb{T}_{k}\left(N^{+} p^{r}\right)_{E}^{N^{-} \text {-new }}, \\
\mathbb{T}_{k}^{N^{-}}\left(N^{+}, p^{r}\right)_{E} & \simeq \mathbb{T}_{k}\left(N^{+}, p^{r}\right)_{E}^{N^{-} \text {-new }}
\end{aligned}
$$

and (non-canonical) isomorphisms of Hecke modules

$$
\begin{aligned}
S_{k}^{N^{-}}\left(N^{+} p^{r}, E\right) & \simeq S_{k}\left(N p^{r}, E\right)^{N^{-} \text {-new }}, \\
S_{k}^{N^{-}}\left(N^{+}, p^{r}, E\right) & \simeq S_{k}\left(N, p^{r}, E\right)^{N^{-} \text {-new }}
\end{aligned}
$$

as $\mathbb{T}_{k}^{N^{-}}\left(N^{+} p^{r}\right)_{E}$-modules and $\mathbb{T}_{k}^{N^{-}}\left(N^{+}, p^{r}\right)_{E}$-modules, respectively. For a classical modular form $f_{k}$, we denote the corresponding quaternionic form by $\phi_{f_{k}}$.

Remark 6.4 (on Theorem 6.3). See also [BD6, Theorem 2.4] for $r=$ 0,1 cases. From this case, one can deduce the general result without any serious difficulty as in $[\mathbf{L V 2}, \S 3.3]$.

6.2. Overconvergent quaternionic forms and control theorems. We mainly follow [PS1]. See also [WXZ, §3] and [LWX, §2]. Let $D_{k}$ be either $\mathscr{D}_{k}$ or $\mathbf{D}_{k}$.

Definition 6.5 (Overconvergent quaternionic forms).

(1) A continuous function $\Phi_{k}: B^{\times} \backslash \widehat{B}^{\times} / \widehat{R}^{(p), \times} \rightarrow D_{k}$ is a $D_{k}$-valued overconvergent quaternionic form of discriminant $N^{-}$, level $N^{+} p^{r}$, and weight $k$ if $\Phi_{k}$ satisfies the following transformation property:

$$
\Phi_{k}(\alpha b u)=\widetilde{\rho}_{k}\left(u_{p}^{-1}\right) \circ \Phi_{k}(b),
$$

where $\alpha \in B^{\times}$and $u \in \widehat{R}_{r}^{\times}$, and $u_{p}$ is the $p$-part of $u$.

(2) The space of such overconvergent quaternionic forms is written as $S^{N^{-}}\left(N^{+} p^{r}, D_{k}\right)$. 
(3) More generally, for a $\Sigma_{0}(p)$-module $A$, we similarly define $A$-valued quaternionic forms and denote the space of such forms by $S^{N^{-}}\left(N^{+} p^{r}, A\right)$ and its variants.

Using the specialization map as in $§ 5.2$, we give an explicit relation between classical and overconvergent quaternionic forms for the noncritical slope case. Let $S^{(<k-1)}$ be the submodule of a Hecke module $S$ of slope $<k-1$ and $\mathbb{T}_{k}^{N^{-}}\left(N^{+} p\right)_{E}^{(<k-1)}$ be the Hecke algebra acting faithfully on the subspace of the forms of slope less than $k-1$.

Theorem 6.6 (Control theorem). There exist $\mathbb{T}_{k}^{N^{-}}\left(N^{+} p\right)_{E}^{(<k-1)}$-equivariant isomorphisms

$$
\begin{aligned}
S^{N^{-}}\left(N^{+} p, \mathscr{D}_{k}\right)^{(<k-1)} & \simeq S^{N^{-}}\left(N^{+} p, \mathbf{D}_{k}\right)^{(<k-1)} \\
& \simeq S_{k}^{N^{-}}\left(N^{+} p, E\right)^{(<k-1)}
\end{aligned}
$$

where the first map is induced from the natural inclusion between distributions and the second map is induced from the specialization map $\mathrm{sp}_{k}$.

For the first isomorphism, see [PS2, Lemma 5.3]. We prove the second isomorphism in $\S 6.5 .2$.

\subsection{Integral normalizations and integral refinement of control} theorems. We introduce an optimal integral normalizations of classical and overconvergent quaternionic forms. These will be used for the slope zero case.

Choice 6.7 (of the "explicit" integral normalizations). Note that all the choices implies that nonvanishing of the form modulo $\varpi$.

(1) If $\Phi_{k} \in S^{N^{-}}\left(N^{+} p, \mathscr{D}_{k}(\mathcal{O})\right)^{(0)}$, then we normalize that the values of $\Phi_{k}$ lie in $\mathscr{D}_{k}(\mathcal{O})$ but not in $\varpi \mathscr{D}_{k}(\mathcal{O})$.

(2) If $\phi_{k} \in S_{k}^{N^{-}}\left(N^{+} p, \mathcal{O}\right)^{(0)}$, then we normalize that the values of $\phi_{k}$ lie in $L_{k}(\mathcal{O})=\operatorname{Sym}^{k-2}(\mathcal{O})$ but not in $\varpi \operatorname{Sym}^{k-2}(\mathcal{O})$.

Choice (1) and (2) are compatible under the specialization map $\mathrm{sp}_{k}$.

Let $S^{(0)}$ be the slope zero submodule of a Hecke module $S$ and $\mathbb{T}_{k}^{N^{-}}\left(N^{+} p\right)^{(0)}$ be the slope zero quotient of $\mathbb{T}_{k}^{N^{-}}\left(N^{+} p\right)$.

Corollary 6.8 (Integral refinement of Theorem 6.6). There exists a $\mathbb{T}_{k}^{N^{-}}\left(N^{+} p\right)^{(0)}$-equivariant isomorphism

$$
S^{N^{-}}\left(N^{+} p, \mathscr{D}_{k}(\mathcal{O})\right)^{(0)} \simeq S_{k}^{N^{-}}\left(N^{+} p, \mathcal{O}\right)^{(0)} .
$$

Remark 6.9. This is a quaternionic analogue of [DHH+, Lemma 6.4]. Note that this explicit integral normalization is not compatible with the 
integral normalization for Hida theory. See [DHH+, Lemma 6.3] for the other integral normalization, which is more relevant to the integral Hida theory. We call the other normalization by the "canonical" integral normalization. These two integral normalizations coincide if $k-1<p$. See the proof of $[\mathbf{D H H}+$, Theorem 6.8].

6.4. A cohomological interpretation. We give a cohomological interpretation of the space of quaternionic forms adapting the approach of completed cohomology à la Emerton with the "trivial" spectral sequence. See [Eme1, (3.2)]. We expect that the explicit Gross points plays the role of the functional on the completed cohomology whose values are (one half of) anticyclotomic $p$-adic $L$-functions as the cycle $(0)-(i \infty) \in \operatorname{Div}^{0}\left(\mathbb{P}^{1}(\mathbb{Q})\right)$ plays the same role on the completed cohomology for $\mathrm{GL}_{2 / \mathbb{Q}}$ to produce cyclotomic $p$-adic $L$-functions. This idea comes from Emerton's comment when the author gave a talk at University of Chicago.

We recall the $\Gamma_{0}\left(p^{\infty}\right)$-variant of completed cohomology for quaternion algebras.

Definition 6.10 (Completed cohomology for quaternion algebras).

$$
\widetilde{\mathrm{H}}^{0}\left(N^{+} p^{\infty}\right):=\underbrace{\lim }_{s} \underset{r}{\lim } \mathrm{H}^{0}\left(B^{\times} \backslash \widehat{B}^{\times} / \widehat{R}_{N^{+} p^{r}}^{\times}, \mathcal{O} / \varpi^{s} \mathcal{O}\right) \text {. }
$$

Let $L_{k, \mathcal{O}}=L_{k}(\mathcal{O})=\operatorname{Sym}^{k-2}\left(\mathcal{O}^{2}\right)$ equipped with a continuous action of an open subgroup $\left(R_{r} \otimes_{\mathbb{Z}} \mathbb{Z}_{p}\right)^{\times}$of $B_{p}^{\times} \simeq \mathrm{GL}_{2}\left(\mathbb{Q}_{p}\right)$. We define the associated $p$-adic local system $\mathscr{L}_{k, \mathcal{O}}=\mathscr{L}_{k}(\mathcal{O})$ on Hida variety $X_{N^{+} p^{r}}^{N^{-}}$as follows:

$$
\mathscr{L}_{k, \mathcal{O}}:=\mathscr{L}_{k}(\mathcal{O}):=B^{\times} \backslash\left(\left(\widehat{B}^{\times} / \widehat{R}_{N^{+} p^{r}}^{(p), \times}\right) \times L_{k, \mathcal{O}}\right) / R_{N^{+} p^{r}, p}^{\times}
$$

For a more detailed description of the local system, see [Eme1, Definition 2.2.3] and [Eme2, 2.1.3]. Then the trivial Hochschild-Serre spectral sequence shows

$$
\operatorname{Hom}_{R_{N^{+} p^{r}, p}^{\times}}\left(L_{k, \mathcal{O}}^{\vee}, \widetilde{\mathrm{H}}^{0}\right) \simeq \mathrm{H}^{0}\left(B^{\times} \backslash \widehat{B}^{\times} / \widehat{R}_{N^{+} p^{r}}^{\times}, \mathscr{L}_{k, \mathcal{O}}\right),
$$

where $L_{k, \mathcal{O}}^{\vee}=\operatorname{Hom}\left(L_{k, \mathcal{O}}, \mathcal{O}\right)$ as in [Eme2, 2.1.3]. Dualizing the first term, we have

$$
\begin{aligned}
\operatorname{Hom}_{R_{N^{+} p^{r}, p}^{\times}}\left(L_{k, \mathcal{O}}^{\vee}, \widetilde{\mathrm{H}}^{0}\right) & \simeq \operatorname{Hom}_{R_{N^{+} p^{r}, p}}\left(\widetilde{\mathrm{H}}_{0}, L_{k, \mathcal{O}}\right) \\
= & \mathrm{H}^{0}\left(R_{N^{+} p^{r}, p}^{\times}, \operatorname{Hom}\left(\widetilde{\mathrm{H}}_{0}, L_{k, \mathcal{O}}\right)\right)=S_{k}^{N^{-}}\left(N^{+} p^{r}, \mathcal{O}\right),
\end{aligned}
$$

where $\widetilde{\mathrm{H}}_{0}$ is the $\mathcal{O}$-dual of $\widetilde{\mathrm{H}}^{0}$. The first isomorphism comes from the fact that $L_{k, \mathcal{O}}$ is a torsion-free $\mathcal{O}$-module of finite rank and $\widetilde{\mathrm{H}}_{0}$ is also a torsion-free $\mathcal{O}$-module. 
6.5. Proof of control theorems. The goal of this subsection is to prove Theorem 6.6 and Corollary 6.8. We follow the strategy of M. Greenberg [Gre, \$4] very closely, which studies the case of modular symbols. Note that the proof is almost identical due to Greenberg's "geometry free" approach. Another virtue of this approach is that it is easy to see the integral nature for the slope zero subspace (Corollary 6.8). See also [Buz1, Proposition 4] for another proof. Buzzard's approach seems more adaptable with the setting of eigenvarieties as in [Buz2].

6.5.1. Preliminaries on distribution modules. Let $\mathbf{D}_{k}(\mathcal{O})=\{\mu \in$ $\mathbf{D}_{k}: \mu\left(z^{n}\right) \in \mathcal{O}$ for all $\left.n \geq 0\right\}$ and it is known that $\mathbf{D}_{k}(\mathcal{O})$ is a $\Sigma_{0}(p)$-stable submodule of $\mathbf{D}_{k}$.

Lemma 6.11 ([Gre, Lemma 1]). Let $\mu \in \mathbf{D}_{k}$. Then the moments $\mu\left(z^{n}\right)$ of $\mu$ are uniformly bounded. Consequently, we have

$$
\mathbf{D}_{k} \simeq \mathbf{D}_{k}(\mathcal{O}) \otimes_{\mathcal{O}} E \text {. }
$$

Define the filtration of $\mathbf{D}_{k}(\mathcal{O})$ as follows:

$\mathrm{Fil}^{0} \mathbf{D}_{k}(\mathcal{O}):=\left\{\mu \in \mathbf{D}_{k}(\mathcal{O}): \mu\left(z^{i}\right)=0\right.$ for all $\left.i=0, \ldots k-2\right\}$,

$\mathrm{Fil}^{m} \mathbf{D}_{k}(\mathcal{O}):=\left\{\mu \in \mathrm{Fil}^{0} \mathbf{D}_{k}(\mathcal{O}): \mu\left(z^{k-2+j}\right) \in \varpi^{m-j+1} \mathcal{O}\right.$ for all $\left.j=1, \ldots m\right\}$

for $m \geq 1$.

Lemma 6.12 ([Gre, Lemma 2]). The submodule $\mathrm{Fil}^{m} \mathbf{D}_{k}(\mathcal{O})$ is $\Sigma_{0}(p)$ stable, for each $m \geq 0$.

Consider the quotients

$$
A^{m} \mathbf{D}_{k}(\mathcal{O}):=\mathbf{D}_{k}(\mathcal{O}) / \mathrm{Fil}^{m} \mathbf{D}_{k}(\mathcal{O})
$$

for all $m \geq 0$. We call $A^{m} \mathbf{D}_{k}(\mathcal{O})$ the $m$-th approximation to the module $\mathbf{D}_{k}(\mathcal{O})$. Note that $L_{k}(\mathcal{O}) \simeq A^{0} \mathbf{D}_{k}(\mathcal{O})$.

Lemma 6.13 ([Gre, Lemma 8]).

$$
S^{N^{-}}\left(N^{+} p, \mathbf{D}_{k}\right) \simeq S^{N^{-}}\left(N^{+} p, \mathbf{D}_{k}(\mathcal{O})\right) \otimes_{\mathcal{O}} E .
$$

Let $m \in L_{k}$ and let $\mu$ be the unique preimage of $m$ under $\operatorname{sp}_{k}$ satisfying $\mu\left(z^{j}\right)=0$ for $j>k-2$. We define the $j$-th moment of $m$ by $m\left(z^{j}\right):=\mu\left(z^{j}\right)$.

The specialization map $\mathrm{sp}_{k}$ naturally induces the Hecke-equivariant map between the spaces of quaternionic forms

$$
\mathrm{sp}_{k, *}: S^{N^{-}}\left(N^{+} p, \mathbf{D}_{k}\right) \longrightarrow S_{k}^{N^{-}}\left(N^{+} p, E\right) .
$$

Consider two natural projections

$$
\operatorname{sp}_{k}^{m}: \mathbf{D}_{k}(\mathcal{O}) \longrightarrow A^{m} \mathbf{D}_{k}(\mathcal{O}), \quad \operatorname{sp}_{k}^{m+1, m}: A^{m+1} \mathbf{D}_{k}(\mathcal{O}) \longrightarrow A^{m} \mathbf{D}_{k}(\mathcal{O}) .
$$

Then the induced maps $\operatorname{sp}_{k, *}^{m}$ and $\operatorname{sp}_{k, *}^{m+1, m}$ on the spaces of quaternionic forms are also $U_{p}$-equivariant. 


\subsubsection{Lifting and control theorems: Proof of Theorem 6.6 and}

Corollary 6.8. Most of the argument does not concern the domain, so the proofs are identical for the case of modular symbols except the convention of group actions.

Let $\alpha=\alpha_{p}\left(f_{k}\right)$ and $h=\operatorname{ord}_{p}(\alpha)$ for convenience. Set

$$
L_{k}^{\alpha}(\mathcal{O}):=\left\{m \in L_{k}(\mathcal{O}): m\left(z^{i}\right) \in \varpi^{h-e \cdot i} \mathcal{O}, 0 \leq i \leq\lfloor h / e\rfloor\right\},
$$

where $e$ is the ramification index of $E / \mathbb{Q}_{p}$ and $\lfloor\cdot\rfloor$ is the floor function. Remark 6.14.

(1) It is known that $L_{k}^{\alpha}(\mathcal{O})$ is $\Sigma_{0}(p)$-stable.

(2) $L_{k}^{\alpha}(\mathcal{O})=L_{k}(\mathcal{O})$ if $h=0$, i.e. slope zero.

Note that $L_{k}^{\alpha}(\mathcal{O}) \otimes_{\mathcal{O}} E=L_{k}(E)$ for any $\alpha$.

Let $\phi^{0} \in S_{2}^{N^{-}}\left(N^{+} p, E\right)$ be an eigenform with $U_{p^{-}}$-eigenvalue $\alpha$ in $E$ of slope strictly less than $k-1$. Assume that $\phi^{0}$ is normalized, i.e. $\phi^{0} \in$ $S_{2}^{N^{-}}\left(N^{+} p, \mathcal{O}\right)$.

Lemma 6.15 ([Gre, Lemma 11]).

(1) Let $\mu \in \mathbf{D}_{k}(\mathcal{O})$ be such that $\operatorname{sp}_{k}(\mu) \in L_{k}^{\alpha}(\mathcal{O})$. Then

$$
\left(\begin{array}{cc}
p & a \\
0 & 1
\end{array}\right) \circ \mu \in \alpha \mathbf{D}_{k}(\mathcal{O})
$$

(2) Let $\mu \in \mathrm{Fil}^{m} \mathbf{D}_{k}(\mathcal{O})$. Then

$$
\left(\begin{array}{ll}
p & a \\
0 & 1
\end{array}\right) \circ \mu \in \alpha \mathrm{Fil}^{m+1} \mathbf{D}_{k}(\mathcal{O}) .
$$

Assume the existence of a lift $\phi^{m}$ of $\phi^{0}$ to $S^{N^{-}}\left(N^{+} p, A^{m} \mathbf{D}_{k}(\mathcal{O})\right)$ such that $\phi^{m}$ is also a $U_{p}$-eigenform with eigenvalue $\alpha$. Choose an arbitrary lift $\Phi$ of $\phi^{m}$ to an element of $\operatorname{Hom}\left(B^{\times} \backslash \widehat{B}^{\times} / \widehat{R}^{(p), \times}, \mathbf{D}_{k}(\mathcal{O})\right)$. Since $\Phi$ is also a lift of $\phi^{0}$, Lemma 6.15(1) implies that

$$
\left(\frac{1}{\alpha} \cdot U_{p}\right) \Phi \in \operatorname{Hom}\left(\mathbb{Z}\left[B^{\times} \backslash \widehat{B}^{\times} / \widehat{R}^{(p), \times}\right], \mathbf{D}_{k}(\mathcal{O})\right) .
$$

Now we define the one step lifting $\phi^{m+1}$ by

$$
\phi^{m+1}:=\operatorname{sp}_{k, *}^{m+1}\left(\left(\frac{1}{\alpha} \cdot U_{p}\right) \Phi\right) \in \operatorname{Hom}\left(\mathbb{Z}\left[B^{\times} \backslash \widehat{B}^{\times} / \widehat{R}^{(p), \times}\right], A^{m+1} \mathbf{D}_{k}(\mathcal{O})\right) .
$$

The $U_{p}$-equivariance of the projection maps together with the relation $\operatorname{sp}_{k}^{m}=\operatorname{sp}_{k}^{m+1, m} \circ \operatorname{sp}_{k}^{m+1}$ implies that $\phi^{m}=\operatorname{sp}_{k, *}^{m+1, m}\left(\phi^{m+1}\right)$.

Lemma 6.16 ([Gre, Claim 1]). The lifted form

$$
\phi^{m+1} \in \operatorname{Hom}\left(\mathbb{Z}\left[B^{\times} \backslash \widehat{B}^{\times} / \widehat{R}^{(p), \times}\right], A^{m+1} \mathbf{D}_{k}(\mathcal{O})\right)
$$

is independent of the choice of lift $\Phi$ used in the construction. 
Proof: The claim immediately follows from Lemma 6.15(2).

Remark 6.17. We do not need to have an analogue of [Gre, Claim 2] since it concerns the special property of the fundamental domain of modular symbols.

The following lemma says that the lift is also $R_{N^{+} p, p}^{\times}$-equivariant.

Lemma 6.18 ([Gre, Claim 3]). The lifted form $\phi^{m+1}$ is $R_{N^{+} p, p}^{\times}$-invariant. In other words,

$$
\gamma \circ \phi^{m+1}=\phi^{m+1}
$$

where $\gamma \in R_{N^{+} p, p}^{\times}$.

Proof: It is a standard computation with help of Lemma 6.16.

The following lemma directly follows from the $\Sigma_{0}(p)$-equivariance of $\mathrm{sp}_{k, *}^{m+1}$.

Lemma 6.19. $\phi^{m+1}$ is a $U_{p}$-eigenform with eigenvalue $\alpha$.

Lemma 6.16, Lemma 6.18, and Lemma 6.19 directly imply the following proposition.

Proposition 6.20 ([Gre, Proposition 12]). The lifted form $\phi^{m+1} \in$ $S^{N^{-}}\left(N^{+} p, A^{m+1} \mathbf{D}_{k}(\mathcal{O})\right)$ is well-defined and independent of the choice of lift $\Phi$ used in the construction. Moreover, $U_{p} \phi^{m+1}=\alpha \phi^{m+1}$ in $S^{N^{-}}\left(N^{+} p, A^{m+1} \mathbf{D}_{k}(\mathcal{O})\right)$.

In order prove Theorem 6.6 , it suffices to prove

$$
S^{N^{-}}\left(N^{+} p, \mathbf{D}_{k}\right)^{U_{p}=\alpha} \simeq S_{k}^{N^{-}}\left(N^{+} p, E\right)^{U_{p}=\alpha}
$$

for each $\alpha$ with $\operatorname{ord}_{p}(\alpha)<k-1$. Also, due to Remark 6.14(2), the following theorem implies Corollary 6.8 immediately.

Theorem 6.21 (Analogue of [Gre, Theorem 9]). Let $\alpha \in E$ be an $U_{p^{-}}$ eigenvalue acting on $S_{k}^{N^{-}}\left(N^{+} p, E\right)$ with noncritical slope $h=\operatorname{ord}_{p}(\alpha)<$ $k-1$. Then the specialization map induces an Hecke-equivariant isomorphism

$$
\operatorname{sp}_{k, *}: S^{N^{-}}\left(N^{+} p, \mathbf{D}_{k}(E)\right)^{U_{p}=\alpha} \longrightarrow S_{k}^{N^{-}}\left(N^{+} p, E\right)^{U_{p}=\alpha} .
$$

Proof: A proof can be taken verbatim from that of [Gre, Theorem 9]. We just remark that Lemma 6.13 and Lemma 6.15(2) are used to prove the injectivity, and Proposition 6.20 is used to prove the surjectivity. 


\section{Construction of $p$-adic $L$-functions}

The goal of this section is to give an overconvergent construction of anticyclotomic $p$-adic $L$-functions as admissible distributions and reconstruct the corresponding theta elements from the distributions.

Let $h<k-1$. We briefly recall the $h$-admissibility of distributions on locally polynomials on $\mathbb{Z}_{p}$, and the work of Amice-Vélu $[\mathbf{A V}]$ and Višik [Viš] (Theorem 7.2) on the lifting of $h$-admissible distributions on locally polynomial functions on $\Gamma_{\infty} \simeq \mathbb{Z}_{p}$ of degree $\leq k-2$, to locally analytic distributions on $\Gamma_{\infty}$. Then we explicitly define the $h$-admissible distribution on locally polynomial functions on $\Gamma_{\infty}$ of degree $\leq k-2$ in terms of the values of quaternionic forms. Applying the lifting result, the distribution extends to a locally analytic distribution on $\Gamma_{\infty}$.

7.0.1. Preliminaries on distributions. We recall the unique lifting of $h$-admissible distributions on locally polynomial functions of degree $\leq$ $k-2$ to locally analytic distributions. See [Viš, §1.3] and [Pol1, §2.1] for detail.

Let $\mathcal{C}^{h}\left(\mathbb{Z}_{p}\right)$ be the space of $\mathbb{C}_{p}$-valued functions on $\mathbb{Z}_{p}$ which are locally polynomials of degree $\leq h$.

Definition 7.1 ( $h$-admissible distributions on locally polynomials). An $h$-admissible distribution $\mu$ on $\mathbb{Z}_{p}$ is a $\mathbb{C}_{p}$-linear map from $\mathcal{C}^{h}\left(\mathbb{Z}_{p}\right)$ to $\mathbb{C}_{p}$ such that

$$
\sup _{a \in \mathbb{Z}_{p}}\left|\mu\left((z-a)^{i} \cdot \mathbf{1}_{a+p^{n} \mathbb{Z}_{p}}(z)\right)\right|
$$

is $O\left(p^{n(h-i)}\right)$ for $0 \leq i \leq h$.

Let $\mathscr{D}$ be the algebra of locally analytic distributions on $\mathbb{Z}_{p}$ with convolution product $*$ but forgetting the weight $k$ action of $\Sigma_{0}(p)$.

Theorem 7.2 (Amice-Vélu, Višik). Let $\mu$ be an h-admissible distribution on locally polynomial functions on $\mathbb{Z}_{p}$ of degree less than or equal to $k-2$. Then $\mu$ extends uniquely to a distribution on locally analytic functions on $\mathbb{Z}_{p}$, i.e. $\mu \in \mathscr{D}$.

Proof: See [AV, Proposition II.2.4], [Viš, Lemma 2.10 and Theorem 3.3], [MTT, Theorem in §11], and [Ste, (6.5) Corollary] for detail. Note that the original statement is given with distributions on $\mathbb{Z}_{p}^{\times}$rather than on $\mathbb{Z}_{p}$. See [Viš, $\S 1.8$ and $\left.\S 2.4\right]$ for the modification.

7.1. The distribution. Let $f_{k} \in S_{k}\left(\Gamma_{0}(N p)\right)$ be a $p$-stabilized newform of non-critical slope and $\phi_{f_{k}}$ be the associated integrally normalized quaternionic form in $S_{k}^{N^{-}}\left(N^{+} p, \mathcal{O}\right)^{(<k-1)}$ as in $\S 6.3$.

Proposition 7.3 ([PS1, Lemma 6.2]). All the values of $\Phi_{f_{k}}$ are $(k-1)$ admissible distributions. 
From now on, we explicitly determine the distribution $\Phi_{f_{k}}\left(\varsigma^{(1)}\right)$, which is one half of the $p$-adic $L$-function.

Definition 7.4 (The distribution).

$$
\mu_{f_{k}, K_{\infty}}:=\Phi_{f_{k}}\left(\varsigma^{(1)}\right) \text {. }
$$

Notation 7.5. Let $W_{n}=\left(\Gamma_{\infty}\right)^{p^{n}} \subseteq \Gamma_{\infty}$ be the unique subgroup of $\Gamma_{\infty}$ of index $p^{n}$. For $a \in \mathbb{Z} / p^{n} \mathbb{Z}$, there exists a unique $\xi_{a} \in \Gamma_{n}=\Gamma_{\infty} / W_{n}$ such that

$$
x_{n}\left(\xi_{a}\right)=\varsigma^{(1)} \cdot\left(\begin{array}{cc}
p^{n} & a \\
0 & 1
\end{array}\right) .
$$

Then $\xi_{a} W_{n}$ is (non-canonically but explicitly) identified with $a+p^{n} \mathbb{Z}_{p}$ in $\Gamma_{\infty} \simeq \mathbb{Z}_{p}$.

Due to Theorem 7.2 and Proposition 7.3, in order to determine the distribution explicitly, it suffices to compute the values

$$
\Phi_{f_{k}}\left(\varsigma^{(1)}\right)\left(z^{j} \cdot \mathbf{1}_{\xi_{a} W_{n}}\right)
$$

explicitly for all $n \geq 1, a \in \mathbb{Z} / p^{n} \mathbb{Z}$, and $j=0, \ldots, k-2$.

Definition 7.6 (The $j$-th component of an quaternionic form). We define the $j$-th component $\phi_{f_{k}}^{[j]}$ of $\phi_{f_{k}}$ by the composition

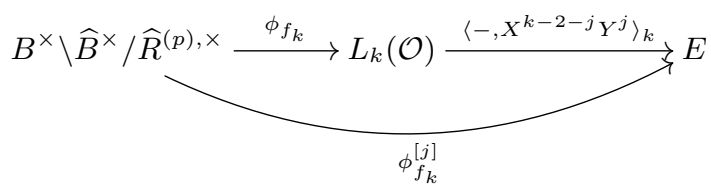

for $j=0, \ldots, k-2$.

First, we compare the evaluation of overconvergent quaternionic forms and the specialization map, which describe the "total measure".

Lemma 7.7 (on the comparison of the total measure). Given any $b \in$ $B^{\times} \backslash \widehat{B}^{\times} / \widehat{R}^{(p), \times}$, we have

$$
\Phi_{f_{k}}(b)\left(z^{j}\right)=\phi_{f_{k}}^{[j]}(b)
$$

for $j=0, \ldots k-2$.

Proof: Recall the specialization map

$$
\operatorname{sp}_{k}\left(\Phi_{f_{k}}(b)\right)=\int_{\mathbb{Z}_{p}}(Y-z X)^{k-2} d \Phi_{f_{k}}(b)(z) .
$$

Also, the control theorem (Theorem 6.6) implies that

$$
\begin{aligned}
\operatorname{sp}_{k, *}\left(\Phi_{f_{k}}\right)(b) & =\operatorname{sp}_{k}\left(\Phi_{f_{k}}(b)\right) \\
& =\phi_{f_{k}}(b) .
\end{aligned}
$$


Thus, we have

$$
\phi_{f_{k}}(b)=\int_{\mathbb{Z}_{p}}(Y-z X)^{k-2} d \Phi_{f_{k}}(b)(z) \in L_{k} .
$$

Pairing with $\left\langle-, X^{k-2-j} Y^{j}\right\rangle_{k}$ as in $\S 5.1 .2$, we have the following equality of the total measure.

$$
\int_{\mathbb{Z}_{p}} z^{j} d \Phi_{f_{k}}(b)(z)=\phi_{f_{k}}^{[j]}(b)
$$

for all $j=0, \ldots, k-2$.

Now we compute all the values following [PS1, Proposition 6.3]. Since $\Phi_{f_{k}}$ is an $U_{p}$-eigenform with eigenvalue $\alpha$, we have

$$
\begin{aligned}
\Phi_{f_{k}}\left(\varsigma^{(1)}\right) & =\alpha^{-n}\left(U_{p}^{n} \Phi_{f_{k}}\right)\left(\varsigma^{(1)}\right) \\
& =\alpha^{-n} \sum_{b=0}^{p^{n}-1}\left(\begin{array}{cc}
p^{n} & b \\
0 & 1
\end{array}\right) \circ\left(\Phi_{f_{k}}\left(\varsigma^{(1)} \cdot\left(\begin{array}{cc}
p^{n} & b \\
0 & 1
\end{array}\right)\right)\right) \\
& =\alpha^{-n} \sum_{b=0}^{p^{n}-1}\left(\begin{array}{ll}
p^{n} & b \\
0 & 1
\end{array}\right) \circ\left(\Phi_{f_{k}}\left(x_{n}\left(\xi_{b}\right)\right)\right) .
\end{aligned}
$$

For any distribution $\mu \in \mathscr{D}_{k}$, the support of $\left(\begin{array}{cc}p^{n} & a \\ 0 & 1\end{array}\right) \circ \mu$ is contained in $a+p^{n} \mathbb{Z}_{p}$. Thus, for $0 \leq j \leq k-2$, we have

$$
\begin{aligned}
\Phi_{f_{k}}\left(\varsigma^{(1)}\right)\left(z^{j} \cdot \mathbf{1}_{a+p^{n} \mathbb{Z}_{p}}(z)\right) \\
=\alpha^{-n} \cdot\left(U_{p}^{n} \Phi_{f_{k}}\right)\left(\varsigma^{(1)}\right)\left(z^{j} \cdot \mathbf{1}_{a+p^{n} \mathbb{Z}_{p}}(z)\right) \\
=\alpha^{-n} \cdot \sum_{b=0}^{p^{n}-1}\left(\begin{array}{cc}
p^{n} & b \\
0 & 1
\end{array}\right) \circ\left(\Phi_{f_{k}}\left(x_{n}\left(\xi_{b}\right)\right)\right)\left(z^{j} \cdot \mathbf{1}_{a+p^{n} \mathbb{Z}_{p}}(z)\right) \\
=\alpha^{-n} \cdot \sum_{b=0}^{p^{n}-1}\left(\Phi_{f_{k}}\left(x_{n}\left(\xi_{b}\right)\right)\right)\left(\left(p^{n} z+b\right)^{j} \cdot \mathbf{1}_{a+p^{n} \mathbb{Z}_{p}}\left(p^{n} z+b\right)\right) \\
=\alpha^{-n} \cdot \Phi_{f_{k}}\left(x_{n}\left(\xi_{a}\right)\right)\left(\left(p^{n} z+a\right)^{j}\right) \\
=\alpha^{-n} \cdot \sum_{i=0}^{j} \Phi_{f_{k}}\left(x_{n}\left(\xi_{a}\right)\right)\left(\left(\begin{array}{l}
j \\
i
\end{array}\right) \cdot\left(p^{n} z\right)^{i} \cdot a^{j-i}\right) \\
=\sum_{i=0}^{j}\left(\frac{p^{i}}{\alpha}\right)^{n} \cdot \phi_{f_{k}}^{[i]}\left(x_{n}\left(\xi_{a}\right)\right) \cdot a^{j-i},
\end{aligned}
$$


by Lemma 7.7 . Thus, the distribution $\Phi_{f_{k}}\left(\varsigma^{(1)}\right)$ is completely determined. Also, for $j \geq 1$, we have

$$
\Phi_{f_{k}}\left(\varsigma^{(1)}\right)\left(\left(z^{j}-a \cdot z^{j-1}\right) \cdot \mathbf{1}_{a+p^{n} \mathbb{Z}_{p}}(z)\right)=\left(\frac{p^{j}}{\alpha}\right)^{n} \cdot \phi_{f_{k}}^{[j]}\left(x_{n}\left(\xi_{a}\right)\right) .
$$

Let $\mu_{f_{k}, K_{\infty}}^{-1}$ is the distribution in $\mathscr{D}$ determined by the values

$$
\mu_{f_{k}, K_{\infty}}^{-1}\left(z^{j} \cdot \mathbf{1}_{a+p^{n} \mathbb{Z}_{p}}\right)=\sum_{i=0}^{j}\left(\frac{p^{i}}{\alpha}\right)^{n} \cdot \phi_{f_{k}}^{[i]}\left(x_{n}^{-1}\left(\xi_{a}\right)\right) \cdot a^{j-i} .
$$

Definition 7.8 ( $p$-adic $L$-functions). The $p$-adic $L$-function $L_{p}\left(K_{\infty}, f\right)$ is defined by the convolution product of distributions

$$
\mu_{f_{k}, K_{\infty}} * \mu_{f_{k}, K_{\infty}}^{-1} \in \mathscr{D} \text {. }
$$

Remark 7.9. This element is well-defined up to a nonzero constant in $\mathcal{O}_{E}^{\times}$ since all the choices defining $\mu_{f_{k}, K_{\infty}}$ and $\mu_{f_{k}, K_{\infty}}^{-1}$ cancel each other.

7.2. Reconstruction of theta elements. In order to obtain the interpolation formula more explicitly, we compare our $p$-adic $L$-functions and those of $[\mathbf{C H}]$ at the level of theta elements (finite layers).

Definition 7.10 (Theta elements). Let

$$
\widetilde{\theta}_{n}\left(f_{k}\right):=\sum_{\xi \in \widetilde{G}_{n}}\left(\frac{1}{\alpha}\right)^{n} \cdot \phi_{f_{k}}^{[0]}\left(x_{n}(\xi)\right) \xi^{-1} \in E\left[\widetilde{G}_{n}\right]
$$

and

$$
\widetilde{\theta}_{n}^{*}\left(f_{k}\right):=\sum_{\xi \in \widetilde{G}_{n}}\left(\frac{1}{\alpha}\right)^{n} \cdot \phi_{f_{k}}^{[0]}\left(x_{n}^{-1}(\xi)\right) \xi^{-1} \in E\left[\widetilde{G}_{n}\right] .
$$

We define the $n$-th theta elements of $f_{k}$ by

$$
\theta_{n}\left(f_{k}\right):=\text { the image of } \theta\left(\widetilde{K}_{n}, f_{k}\right) \text { under the projection } E\left[\widetilde{G}_{n}\right] \rightarrow E\left[\Gamma_{n}\right]
$$

and

$\theta_{n}^{*}\left(f_{k}\right):=$ the image of $\theta^{*}\left(\widetilde{K}_{n}, f_{k}\right)$ under the projection $E\left[\widetilde{G}_{n}\right] \rightarrow E\left[\Gamma_{n}\right]$.

Remark 7.11 (on the well-definedness). Each element is defined only up to multiplication by an element of $\widetilde{G}_{n}$ due to the choices we made. Only the element

$$
L_{p, n}\left(K_{\infty}, f_{k}\right):=\theta_{n}\left(f_{k}\right) \cdot \theta_{n}^{*}\left(f_{k}\right) \in E\left[\Gamma_{n}\right]
$$

is well-defined.

Remark 7.12 (on the boundedness of coefficients). By construction, it is easy to observe that

$$
\theta_{n}\left(f_{k}\right) \in \frac{1}{\alpha^{n}} \mathcal{O}_{E}\left[\Gamma_{n}\right] .
$$

(cf. [CL, Remark 2.5 and Definition 2.6], [CH, Lemma 4.4(1)]). 


\section{The "weak" interpolation formula}

The goal of this section to prove the "weak" interpolation formula for our $p$-adic $L$-functions, indeed $p$-adic theta elements. We use the interpolation formula for complex theta elements in $[\mathbf{C H}]$. Since our $p$-adic theta elements and complex theta elements of $[\mathbf{C H}]$ are only congruent modulo $p^{n}$ at explicit Gross points of conductor $p^{n}$ (Corollary 8.8), the integrally refined interpolation formula (Corollary 8.9) is given only as a congruence formula unless the form is ordinary or of weight two.

Remark 8.1. All the normalizations are slightly different from those of $[\mathbf{C H}]$ mainly due to the normalization of the pairing. The index is also different because $[\mathbf{C H}]$ mainly focus on the central critical $L$-values and we mainly concern the growth of the distribution we defined.

\subsection{Complex quaternionic forms and $p$-adic quaternionic forms.} Using $i_{\mathbb{C}}$, we define the representation

$$
\rho_{k, \infty}: B(\mathbb{R})^{\times} \longrightarrow \mathrm{GL}_{2}(\mathbb{C}) \longrightarrow \operatorname{Aut}_{\mathbb{C}}\left(L_{k}(\mathbb{C})\right) .
$$

Then $\mathbb{C} \cdot X^{i} Y^{k-2-i} \subseteq L_{k}(\mathbb{C})$ or $L_{k}(2-k)(\mathbb{C})$ is the eigenspace on which $\rho_{k, \infty}(t)$ acts with eigenvalue $\bar{t}^{i} \cdot t^{k-2-i}$ or $\rho_{k, \infty}^{*}(t)$, with eigenvalue $\bar{t}^{i-(k-2)} \cdot t^{-i}$ for $t \in(K \otimes \mathbb{R})^{\times}(\mathrm{cf}$. [CH, $\left.\S 2.3]\right)$.

Let $U$ be an open compact subgroup of $\widehat{B}^{\times}$.

Definition 8.2 (Complex quaternionic forms). A function $\mathbf{f}_{k}: \widehat{B}^{\times} \rightarrow$ $L_{k}(\mathbb{C})$ is a complex quaternionic form of weight $k$ and level $U$ if $\mathbf{f}_{k}$ satisfies the transformation property

$$
\mathbf{f}_{k}(\alpha b u)=\rho_{k, \infty}(\alpha) \circ \mathbf{f}_{k}(b),
$$

where $\alpha \in B^{\times}$and $u \in U$.

The space of complex quaternionic forms is denoted by $\mathbf{S}_{k}^{N^{-}}(U, \mathbb{C})$. Then $\mathbf{S}_{k}^{N^{-}}(\mathbb{C}):=\underset{U}{\lim } \mathbf{S}_{k}^{N^{-}}(U, \mathbb{C})$ becomes an admissible representation of $\widehat{B}^{\times}$.

Let $B(\mathbb{A})^{\times}=(B \otimes \mathbb{A})^{\times}$where $\mathbb{A}$ is the ring of adeles over $\mathbb{Q}$. For $\mathbf{f}_{k} \in \mathbf{S}_{k}^{N^{-}}(\mathbb{C})$ and $P(X, Y) \in L_{k}(2-k)(\mathbb{C})$, we define a function $\Psi\left(\mathbf{f}_{k} \otimes\right.$ $P(X, Y)): B^{\times} \backslash B(\mathbb{A})^{\times} \rightarrow \mathbb{C}$ by

$$
\Psi\left(\mathbf{f}_{k} \otimes P(X, Y)\right)(g):=\left\langle\mathbf{f}_{k}\left(g_{f}\right), \rho_{k, \infty}^{*}\left(g_{\infty}\right) \circ P(X, Y)\right\rangle_{k},
$$

where $\rho_{k, \infty}^{*}\left(g_{\infty}\right)=\operatorname{det}^{2-k}\left(g_{\infty}\right) \cdot \rho_{k, \infty}\left(g_{\infty}\right)$ (cf. [CH, (2.11)]).

We define

$$
\rho_{k, p}: B_{p}^{\times} \longrightarrow \operatorname{Aut}_{\mathbb{C}_{p}}\left(L_{k}\left(\mathbb{C}_{p}\right)\right)
$$


by $\rho_{k, p}(g):=\rho_{k} \circ \iota_{p} \circ i_{K}(g)$ for $g \in B_{p}^{\times}$, and define $\rho_{k, p}^{*}(g):=\operatorname{det}^{2-k}(g)$. $\rho_{k, p}(g)$. By $[\mathbf{C H}, \S 4.1]$, we have

$$
\rho_{k, p}(g)=\rho_{k, \infty}(g)
$$

for $g \in B^{\times}$, and

$$
\rho_{k, p}(g)=\rho_{k}\left(\gamma_{p} \cdot i_{p}(g) \cdot \gamma_{p}^{-1}\right)
$$

for $g \in B_{p}^{\times}$, where $\gamma_{p}:=\left(\begin{array}{c}\sqrt{\beta}-\sqrt{\beta \theta} \\ -1\end{array}\right) \in \mathrm{GL}_{2}\left(K_{p}\right)$ and $i_{p}$ is the fixed isomorphism $B_{p} \simeq \mathrm{M}_{2}\left(\mathbb{Q}_{p}\right)$.

Let $A$ be a subring of $\mathbb{C}$ and $\mathbf{S}_{k}^{N^{-}}\left(N^{+} p, A\right) \subseteq \mathbf{S}_{k}^{N^{-}}\left(N^{+} p, \mathbb{C}\right)$ and $S_{k}^{N^{-}}\left(N^{+} p, A\right) \subseteq S_{k}^{N^{-}}\left(N^{+} p, \mathbb{C}_{p}\right)$ (via $\iota_{p}$ and $\iota_{\infty}$ ) be the submodules of $A$-valued forms. If $\frac{1}{p} \in A$, then we have the isomorphism

$$
\begin{aligned}
\mathbf{S}_{k}^{N^{-}}\left(N^{+} p, A\right) & \stackrel{\simeq}{\longrightarrow} S_{k}^{N^{-}}\left(N^{+} p, A\right) \\
\mathbf{f}_{k} & \longmapsto \widehat{\mathbf{f}}_{k}(g)=\phi_{f_{k}}(g):=\rho_{k}\left(\gamma_{p}^{-1}\right) \cdot \rho_{k, p}\left(g_{p}^{-1}\right) \circ \mathbf{f}_{k}(g),
\end{aligned}
$$

where $g \in \widehat{B}^{\times}$and $g_{p}$ is the $p$-part of $g$. Furthermore, with the JacquetLanglands correspondence (Theorem 6.3), we identify

$$
\begin{aligned}
\mathbf{S}_{k}^{N^{-}}\left(N^{+} p, A\right) \stackrel{\simeq}{\longrightarrow} S_{k}^{N^{-}}\left(N^{+} p, A\right) \stackrel{\simeq}{\longleftarrow} S_{k}\left(N^{+} p, A\right)^{N^{-}-\text {new }} \\
\mathbf{f}_{k} \longmapsto \widehat{\mathbf{f}}_{k}=\phi_{f_{k}} \longleftarrow f_{k}
\end{aligned}
$$

up to multiplication by an element of $A^{\times}$.

8.2. Complex theta elements of higher weight. From now on, we assume $\mathbf{f}_{k} \in \mathbf{S}_{k}^{N^{-}}\left(N^{+} p, \mathbb{C}\right)$ is a $p$-stabilized newform with $U_{p^{-}}$-eigenvalue $\alpha$ with non-critical slope (cf. [CH, §3.2]). Let

$$
v_{i}^{*}:=(-1)^{\frac{k-2}{2}} \cdot(-1)^{i} \cdot\left(\begin{array}{c}
k-2 \\
i
\end{array}\right) \cdot \sqrt{\beta}^{k-2-i} \cdot{\sqrt{-D_{K}}}^{k-2} \cdot X^{i} Y^{k-2-i} \in L_{k}(2-k)(\mathbb{C})
$$

for $i=0, \ldots, k-2($ cf. $[\mathbf{C H},(3.1)])$.

Let

$$
\mathbf{f}_{k, i}:=\Psi\left(\mathbf{f}_{k} \otimes v_{i}^{*}\right) .
$$

Let $\widetilde{G}_{n}:=K^{\times} \backslash \widehat{K}^{\times} / \mathcal{O}_{n}^{\times} \cdot \widehat{\mathbb{Q}}^{\times}$be the Galois group of the ring class field of $K$ of conductor $p^{n}$. It coincides with the same notation in $\S 3.2$. Here, $\mathcal{O}_{n}=\mathbb{Z}+p^{n} \mathcal{O}_{K}$. Let $[\cdot]_{n}: \widehat{K}^{\times} \rightarrow \widetilde{G}_{n}$ be the geometrically normalized reciprocity map.

Definition 8.3 (Complex theta elements, [CH, Definition 4.1]). Fix a set $\Xi_{n}$ of representatives of $\widetilde{G}_{n}$ in $K^{\times} \backslash \widehat{K}^{\times}$. We define the $n$-th complex theta element $\Theta_{n}^{[i]}\left(\mathbf{f}_{k}\right)$ of weight $i-\frac{k-2}{2}$ by

$$
\Theta_{n}^{[i]}\left(\mathbf{f}_{k}\right):=\frac{1}{\alpha^{n}} \sum_{a \in \Xi_{n}} \mathbf{f}_{k, i}\left(x_{n}(a)\right) \cdot \iota_{p}^{-1}\left(\bar{a}_{p}^{i-(k-2)} \cdot a_{p}^{-i}\right) \cdot[a]_{n} \in \mathbb{C}\left[\widetilde{G}_{n}\right] .
$$


Remark 8.4. Note that the index and the weight of the complex theta element are different due to Remark 8.1.

\subsection{The interpolation formula for the complex theta elements.}

Definition 8.5. Let $\chi$ be an anticyclotomic Hecke character of conductor $p^{s}$ and weight $\left(i-\frac{k-2}{2}, i+\frac{k-2}{2}\right)$ where $i=1, \ldots k-1$. We define the (central critical twisted) p-adic avatar $\widehat{\chi}$ of $\chi$ by

$$
\widehat{\chi}(a)=\chi(a) \cdot a_{p}^{i} \cdot \bar{a}_{p}^{k-2-i}
$$

with respect to the weight $k$ of $f$ (cf. [CH, Introduction]).

Next we state the interpolation formula for the complex theta element $\Theta_{n}^{[i]}\left(\mathbf{f}_{k}\right)$. Note that the formula is slightly different from the original one, but this is only because of the difference of the normalization.

Theorem 8.6 ([CH, Proposition 4.3]). Suppose that $\chi$ has the conductor $p^{s}$. For every $n \geq \max \{s, 1\}$, we have the interpolation formula

$$
\begin{array}{r}
\widehat{\chi}\left(\Theta_{n}^{[i]}\left(\mathbf{f}_{k}\right)^{2}\right)=(k-2) ! \cdot \frac{L\left(f_{k}, \chi, \frac{k}{2}\right)}{\Omega_{f_{k}, N^{-}}} \cdot e_{p}\left(f_{k}, \chi\right)^{j} \cdot \frac{1}{\alpha^{2 s}} \cdot\left(p^{s} D_{K}\right)^{k-1} \cdot \frac{u_{K}^{2}}{\sqrt{D_{K}}} \\
\cdot \chi\left(\mathfrak{N}^{+}\right) \cdot \epsilon_{p}\left(f_{k}\right),
\end{array}
$$

where

- $\Omega_{f_{k}, N^{-}}$is the Gross period defined in $[\mathbf{C H},(4.3)]$ (cf. [Kim, Appendix]),

- $e_{p}\left(f_{k}, \chi\right)$ is the $p$-adic multiplier defined by

$$
e_{p}\left(f_{k}, \chi\right)= \begin{cases}1 & \text { if } n>0, \\ \left(1-p^{\frac{k-2}{2}} \alpha_{p}\left(f_{k}\right)^{-1}\right)^{2} & \text { if } n=0 \text { and } p \text { splits in } K, \\ 1-p^{k-2} \alpha_{p}\left(f_{k}\right)^{-2} & \text { if } n=0 \text { and } p \text { is inert in } K,\end{cases}
$$

and

$$
j= \begin{cases}1 & \text { if } f_{k} \text { is new at } p \\ 2 & \text { if } f_{k} \text { is old at } p\end{cases}
$$

- $u_{K}^{2}=\#\left(\mathcal{O}_{K}^{\times} / 2\right)$,

- $\mathfrak{N}^{+}$is the ideal of $\mathcal{O}_{K}$ satisfying $N^{+}=\mathfrak{N}^{+} \cdot \overline{\mathfrak{N}^{+}}$in $K$ depending on the orientation of the optimal embedding, and

- $\epsilon_{p}\left(f_{k}\right)$ is the eigenvalue of the Atkin-Lehner involution of $f_{k}$ at $p$.

In $[\mathbf{C H}$, Theorem A] there are certain restrictions on weight $(k-2<$ $p$ ) and slope (slope zero). However, Theorem 8.6 does not have such a restriction. 
8.4. An integral comparison of complex and $p$-adic quaternionic forms. The following proposition plays the key role in the integral connection between complex and $p$-adic theta elements.

Proposition 8.7 ([CH, Lemma 4.4]). Let $A \subset \mathbb{C}_{p}$ be a subring which contains $\mathcal{O}_{E}$ and $\mathcal{O}_{K_{p}}$. For $a \in \widehat{K}^{\times}$, we have

$$
p^{n(k-2)} \cdot \mathbf{f}_{k, i}\left(x_{n}(a)\right) \cdot \bar{a}_{p}^{i-(k-2)} \cdot a_{p}^{-i} \in A,
$$

and

$$
\begin{aligned}
& p^{n(k-2)} \cdot \mathbf{f}_{k, i}\left(x_{n}(a)\right) \cdot \bar{a}_{p}^{i-(k-2)} \cdot a_{p}^{-i} \\
& \equiv(-1)^{\frac{k-2}{2}} \cdot(-1)^{i} \cdot\left(\begin{array}{c}
k-2 \\
i
\end{array}\right) \cdot \sqrt{\beta}^{2-k} \cdot\left\langle\phi_{f_{k}}\left(x_{n}(a)\right), X^{k-2}\right\rangle_{k} \quad\left(\bmod p^{n} A\right)
\end{aligned}
$$

up to multiplication by an element of $A^{\times}$, where $a_{p} \in\left(K \otimes \mathbb{Q}_{p}\right)^{\times}$is the p-part of $a \in \widehat{K}^{\times}$and $\bar{a}_{p}$ is the conjugate of $a_{p}$.

Proof: This is [CH, Lemma 4.4] with change of normalization of the pairing (§5.1.2) and [CL, Remark 2.5].

The following corollary is an immediate consequence.

\section{Corollary 8.8.}

$$
p^{n(k-2)} \cdot \Theta_{n}^{[i]}\left(\mathbf{f}_{k}\right) \equiv(-1)^{\frac{k-2}{2}} \cdot(-1)^{i} \cdot\left(\begin{array}{c}
k-2 \\
i
\end{array}\right) \cdot \sqrt{\beta}^{2-k} \cdot \theta_{n}\left(f_{k}\right)\left(\bmod \frac{p^{n}}{\alpha_{p}^{n}} \mathcal{O}_{E}\right)
$$

up to multiplication by an element of $\mathcal{O}_{E}^{\times}$.

Combining Theorem 8.6 and Corollary 8.8, we have the following interpolation formula.

Corollary 8.9 ("Weak" interpolation formula). Let $\widehat{\chi}$ be a character as in Definition 8.5. Then:

$$
\begin{aligned}
(-1)^{\frac{k-2}{2}} \cdot(-1)^{i} \cdot\left(\begin{array}{c}
k-2 \\
i
\end{array}\right) \cdot \sqrt{\beta}^{2-k} \cdot \widehat{\chi}\left(\theta_{n}\left(f_{k}\right)\right) & \\
\equiv\left(p^{n(k-2)} \cdot(k-2) ! \cdot \frac{L\left(f_{k}, \chi, \frac{k}{2}\right)}{\Omega_{f, N-}} \cdot\right. & e_{p}\left(f_{k}, \chi\right)^{j} \cdot \frac{1}{\alpha^{2 s}} \cdot\left(p^{s} D_{K}\right)^{k-1} \cdot \frac{u_{K}^{2}}{\sqrt{D_{K}}} \\
& \left.\chi\left(\mathfrak{N}^{+}\right) \cdot \epsilon_{p}\left(f_{k}\right)\right)^{1 / 2}\left(\bmod \frac{p^{n}}{\alpha_{p}^{n}} \mathcal{O}_{E}\right)
\end{aligned}
$$

up to multiplication by an element of $\mathcal{O}_{E}^{\times}$. If $f_{k}$ is ordinary or $k=2$, then the congruence becomes equality. 
Remark 8.10. In the "weak" interpolation formula, the congruence immediately becomes equality due to the isomorphism (8.1) if we do not care their integrality, i.e. if we allow they may differ by an element of $E^{\times}$. If the form is ordinary, then the congruence in the weak interpolation formula becomes equality by taking the limit $n \rightarrow \infty$. Then the range of interpolating characters becomes larger, namely the set of locally algebraic $p$-adic characters of weight $(i,-i)$ with $-k / 2<i<k / 2$ as in $[\mathbf{C H}$, Theorem 4.6].

\section{Speculations and questions}

9.1. "Deformation" of explicit Gross points. This is inspired by [Eme1, (4.5)]. We may interpret the explicit Gross point $\varsigma^{(1)}$ as a functional on $S_{k}^{N^{-}}\left(N^{+} p, E\right)^{(<k-1)}$ via the overconvergent construction:

$$
\begin{aligned}
\left(\varsigma^{(1)}\right)_{*}: S_{k}^{N^{-}}\left(N^{+} p, E\right)^{(<k-1)} & \longrightarrow \mathscr{D} \\
\phi_{f_{k}} \longmapsto & \longrightarrow \Phi_{f_{k}}\left(\varsigma^{(1)}\right) .
\end{aligned}
$$

This can be regarded as a functional on a small piece (i.e. fixed weight) of the completed cohomology as in $\S 6.4$ or the corresponding eigenvariety. If we can "patch" this functional on all the weight coherently, the map $\left(\varsigma^{(1)}\right)_{*}$ would extend to the functional on the whole completed cohomology or the corresponding eigenvariety, which may produce two variable anticyclotomic $p$-adic $L$-functions on the eigenvariety. In the sequel paper in preparation, we construct two variable anticyclotomic $p$-adic $L$-functions of Hida families and study their Iwasawa theory.

Furthermore, if we work with geometry of the eigenvariety attached to a definite quaternion algebra (e.g. [Buz2]) or uses a relevant $p$-adic extension of Jacquet-Langlands correspondence (e.g. [HIS]) in more detail, then we may be able to generalize the main result of this article to the critical slope case as in [Bel]. In fact, our setting prevents CM forms; thus, it would be easier than the case of modular symbols.

9.2. Growth of the size of noncommutative class numbers. It seems very interesting if we see a certain Iwasawa-theoretic phenomena in the growth behavior of the size of $B^{\times} \backslash \widehat{B}^{\times} / \widehat{R}_{N^{+} p^{r}}$ as $r \rightarrow \infty$. Then the study of this growth would be regarded as a "noncommutative Iwasawa theory" in a completely different sense. Maybe a $p$-adic variation of the Eichler trace formula [Vig, chapitre III, §5.C] would yield an asymptotic formula like Iwasawa's formula on the $p$-class numbers of the $p$-cyclotomic fields.

9.3. Geometric aspect of quaternionic Hida theory. In [LV1, $§ 2$ and $\S 6]$, Longo and Vigni investigate the geometric aspect of quaternionic 
Hida theory. If we can capture the $\Gamma_{1}\left(p^{r}\right)$ and $\Gamma_{1}\left(p^{\infty}\right)$-level structures in the context of the Bruhat-Tits tree or its suitable coverings, then it may allow us to consider a direct connection of the Bruhat-Tits tree and quaternionic Hida theory. Also, it would naturally explain the relation between geometric Gross points and big Gross points à la LongoVigni $[\mathbf{L V 1}, \S 7]$ in the ordinary case.

9.4. Explicit computation. One may implement an explicit overconvergent algorithm, which is expected to be as effective as one in $[\mathbf{G M}]$, to improve the computation of theta elements given in [BD1, §5.1]. The explicit computation of the quotient graph $[\mathbf{F M}]$ seems helpful to do this at least for the case of weight two forms. In [Grä], P. M. Gräf explicitly computed Teitelbaum $\mathcal{L}$-invariants of $p$-newforms. If one implement the computation of theta elements for not only $p$-newforms but also $p$-stabilized newforms, then it would have many arithmetic applications. For the cyclotomic case, see $[\mathbf{D H H}+]$.

\section{Acknowledgement}

The author thanks Karl Rubin for a year-long discussion on this project. This project has started when the author was a visiting assistant professor at UC Irvine. The author thanks Robert Pollack and Glenn Stevens for the inspiration of this work; Lawrence Yong-uck Chung for helping me to understand Proposition 3.9; Ming-Lun Hsieh for pointing out various technical issues; Chol Park, Sug Woo Shin, and Yiwen Zhou for helpful discussion. Some part of this work is inspired by the talk by Carlos de Vera Piquero at CRM, March 2015. This research is partially supported by Basic Science Research Program through the National Research Foundation of Korea (NRF-2018R1C1B6007009).

\section{References}

[AV] Y. Amice And J. VÉLU, Distributions $p$-adiques associées aux séries de Hecke, in: "Journées Arithmétiques de Bordeaux" (Conf., Univ. Bordeaux, Bordeaux, 1974), Astérisque 24-25, Soc. Math. France, Paris, 1975, pp. 119-131.

[Bel] J. Bellä̈che, Critical p-adic L-functions, Invent. Math. 189(1) (2012), 1-60. DOI : $10.1007 / \mathrm{s} 00222-011-0358-z$.

[BG] F. Bergunde and L. Gehrmann, Leading terms of anticyclotomic Stickelberger elements and $p$-adic periods, Trans. Amer. Math. Soc. 370(9) (2018), 6297-6329. DOI : 10.1090/tran/7120.

[BD1] M. Bertolini And H. Darmon, Heegner points on Mumford-Tate curves, Invent. Math. 126(3) (1996), 413-456. DOI : 10.1007/s002220050105. 
[BD2] M. Bertolini and H. Darmon, Heegner points, $p$-adic $L$-functions, and the Cerednik-Drinfeld uniformization, Invent. Math. 131(3) (1998), 453-491. DOI : $10.1007 / \mathrm{s} 002220050211$.

[BD3] M. Bertolini and H. Darmon, $p$-adic periods, $p$-adic $L$-functions, and the $p$-adic uniformization of Shimura curves, Duke Math. J. 98(2) (1999), 305-334. DOI : 10.1215/S0012-7094-99-09809-5.

[BD4] M. Bertolini and H. Darmon, The $p$-adic $L$-functions of modular elliptic curves, in: "Mathematics Unlimited - 2001 and Beyond", Springer, Berlin, 2001, pp. 109-170. DOI : 10.1007/978-3-642-56478-9.

[BD5] M. Bertolini AND H. DARMOn, Iwasawa's main conjecture for elliptic curves over anticyclotomic $\mathbb{Z}_{p}$-extensions, Ann. of Math. (2) 162(1) (2005), 1-64. DOI : 10.4007/annals .2005.162.1.

[BD6] M. Bertolini And H. Darmon, Hida families and rational points on elliptic curves, Invent. Math. 168(2) (2007), 371-431. DOI : 10.1007/s00222-0070035-4.

[BDI] M. Bertolini, H. Darmon, and A. Iovita, Families of automorphic forms on definite quaternion algebras and Teitelbaum's conjecture, Astérisque $\mathbf{3 3 1}$ (2010), 29-64.

[BDiS] M. Bertolini, H. Darmon, A. Iovita, And M. Spiess, Teitelbaum's exceptional zero conjecture in the anticyclotomic setting, Amer. J. Math. 124(2) (2002), 411-449. DOI : 10.1353/ajm.2002.0009.

[Buz1] K. Buzzard, On p-adic families of automorphic forms, in: "Modular Curves and Abelian Varieties", Progr. Math. 224, Birkhäuser, Basel, 2004, pp. 23-44. DOI : $10.1007 / 978-3-0348-7919-4 \_2$.

[Buz2] K. Buzzard, Eigenvarieties, in: "L-functions and Galois Representations", London Math. Soc. Lecture Note Ser. 320, Cambridge Univ. Press, Cambridge, 2007, pp. 59-120. DOI : 10.1017/СB09780511721267.004.

[CKL] F. Castella, C.-H. Kim, and M. Longo, Variation of anticyclotomic Iwasawa invariants in Hida families, Algebra Number Theory 11(10) (2017), 2339-2368. DOI : 10.2140/ant.2017.11.2339.

[CL] F. Castella And M. Longo, Big Heegner points and special values of $L$-series, Ann. Math. Qué. 40(2) (2016), 303-324. DOI : 10.1007/s40316-0150045-3.

[CH] M. Chida And M.-L. Hsien, Special values of anticyclotomic $L$-functions for modular forms, J. Reine Angew. Math. 741 (2018), 87-131. DOI: 10.1515/crelle-2015-0072.

[CV] C. Cornut and V. Vatsal, Nontriviality of Rankin-Selberg $L$-functions and CM points, in: "L-functions and Galois Representations", London Math. Soc. Lecture Note Ser. 320, Cambridge Univ. Press, Cambridge, 2007, pp. 121-186. DOI : 10.1017/CB09780511721267.005.

[DI] H. DARMON AND A. IOvita, The anticyclotomic main conjecture for elliptic curves at supersingular primes, J. Inst. Math. Jussieu 7(2) (2008), 291-325. DOI : $10.1017 / \mathrm{S} 1474748008000042$.

[DP] H. DARMON AND R. POLlaCK, Efficient calculation of Stark-Heegner points via overconvergent modular symbols, Israel J. Math. 153(1) (2006), 319-354. DOI : $10.1007 / \mathrm{BF} 02771789$.

[DJP] L. De Feo, D. JAO, And J. PlÛt, Towards quantum-resistant cryptosystems from supersingular elliptic curve isogenies, J. Math. Cryptol. 8(3) (2014), 209-247. DOI : 10.1515/jmc-2012-0015. 
[Dep] H. Deppe, $p$-adic $L$-functions of automorphic forms and exceptional zeros, Doc. Math. 21 (2016), 689-734.

[DHh+] E. P. Dummit, M. Hablicsek, R. Harron, L. Jain, R. Pollack, AND D. Ross, Explicit computations of Hida families via overconvergent modular symbols, Res. Number Theory 2, Art. 25 (2016), 54 pp. DOI: $10.1007 / \mathrm{s} 40993-016-0052-8$.

[Eme1] M. EMERTON, On the interpolation of systems of eigenvalues attached to automorphic Hecke eigenforms, Invent. Math. 164(1) (2006), 1-84. DOI: $10.1007 / \mathrm{s} 00222-005-0448-\mathrm{x}$.

[Eme2] M. Emerton, Completed cohomology and the p-adic Langlands program, in: "Proceedings of the International Congress of Mathematicians - Seoul 2014", Vol. II, Kyung Moon Sa, Seoul, 2014, pp. 319-342.

[FM] C. Franc and M. Masdeu, Computing fundamental domains for the Bruhat-Tits tree for $\mathrm{GL}_{2}\left(\mathbf{Q}_{p}\right)$, p-adic automorphic forms, and the canonical embedding of Shimura curves, LMS J. Comput. Math. 17(1) (2014), 1-23. DOI : $10.1112 /$ S1461157013000235.

[Grä] P. M. GRÄF, A control theorem for $p$-adic automorphic forms and Teitelbaum's $\mathcal{L}$-invariant, Preprint (2017). arXiv:1705.02158.

[Gre] M. Greenberg, Lifting modular symbols of non-critical slope, Israel J. Math. 161(1) (2007), 141-155. DOI : 10.1007/s11856-007-0076-9.

[GS] R. Greenberg and G. Stevens, $p$-adic $L$-functions and $p$-adic periods of modular forms, Invent. Math. 111(1) (1993), 407-447. DOI: 10.1007/ BF01231294.

[Gro] B. H. Gross, Heights and the special values of $L$-series, in: "Number Theory" (Montreal, Que., 1985), CMS Conf. Proc. 7, Amer. Math. Soc., Providence, RI, 1987, pp. 115-187.

[GM] X. Guitart and M. Masdeu, Overconvergent cohomology and quaternionic Darmon points, J. Lond. Math. Soc. (2) 90(2) (2014), 495-524. DOI : $10.1112 / \mathrm{jlms} / \mathrm{jdu} 036$.

[HIS] M. Harris, A. Iovita, and G. Stevens, The Jacquet-Langlands correspondence via $\ell$-adic uniformization, Preprint (2010).

[Kim] C.-H. KIM, Anticyclotomic Iwasawa invariants and congruences of modular forms, Asian J. Math. 21(3) (2017), 499-530. DOI: 10.4310/AJM. 2017. v21.n3.a5.

[LWX] R. LiU, D. WAN, AND L. XIAO, The eigencurve over the boundary of weight space, Duke Math. J. 166(9) (2017), 1739-1787. DOI : 10.1215/00127094$0000012 \mathrm{X}$.

[Lon] M. Longo, Anticyclotomic Iwasawa's main conjecture for Hilbert modular forms, Comment. Math. Helv. 87(2) (2012), 303-353. DOI: 10.4171/CMH/ 255.

[LV1] M. Longo And S. Vigni, Quaternion algebras, Heegner points and the arithmetic of Hida families, Manuscripta Math. 135(3-4) (2011), 273-328. DOI : $10.1007 / \mathrm{s} 00229-010-0409-6$.

[LV2] M. Longo AND S. Vigni, A note on control theorems for quaternionic Hida families of modular forms, Int. J. Number Theory 8(6) (2012), 1425-1462. DOI : $10.1142 / \mathrm{S} 1793042112500856$.

[MS] B. Mazur and P. Swinnerton-Dyer, Arithmetic of Weil curves, Invent. Math. 25(1) (1974), 1-61. DOI : 10.1007/BF01389997. 
[MTT] B. Mazur, J. Tate, and J. Teitelbaum, On $p$-adic analogues of the conjectures of Birch and Swinnerton-Dyer, Invent. Math. 84(1) (1986), 1-48. DOI : $10.1007 / \mathrm{BF} 01388731$.

[Mol] S. Molina, Anticyclotomic $p$-adic $l$-functions and the exceptional zero phenomenon, Trans. Amer. Math. Soc. (to appear).

[Pol1] R. Pollack, On the $p$-adic $L$-function of a modular form at a supersingular prime, Duke Math. J. 118(3) (2003), 523-558. DOI: 10.1215/S0012-709403-11835-9.

[Pol2] R. Pollack, Overconvergent modular symbols, in: "Computations with Modular Forms", Contrib. Math. Comput. Sci. 6, Springer, Cham, 2014, pp. 69-105. DOI : 10.1007/978-3-319-03847-6_3.

[PS1] R. Pollack And G. Stevens, Overconvergent modular symbols and $p$-adic $L$-functions, Ann. Sci. Éc. Norm. Supér. (4) 44(1) (2011), 1-42. DOI: 10.24033/asens. 2139 .

[PS2] R. Pollack and G. Stevens, Critical slope $p$-adic $L$-functions, J. Lond. Math. Soc. (2) 87(2) (2013), 428-452. DOI: 10.1112/jlms/jds057.

[Ste] G. Stevens, Rigid analytic modular symbols, Preprint (1994).

[Vig] M.-F. Vignéras, "Arithmétique des algèbres de quaternions", Lecture Notes in Mathematics 800, Springer, Berlin, 1980. DOI: 10.1007/BFb 0091027.

[Viš] M. M. VIŠIK, Non-archimedean measures connected with Dirichlet series, Math. USSR-Sb. 28(2) (1976), 216-228. DOI : 10.1070SM1976v028n02ABEH 001648.

[WXZ] D. WAN, L. XIAO, AND J. Zhang, Slopes of eigencurves over boundary disks, Math. Ann. 369(1-2) (2017), 487-537. DOI : 10.1007/s00208-016-1458-2.

[Yua] H. YuAn, Special value formulae of Rankin-Selberg $L$-functions, Thesis (Ph.D.), University of Pennsylvania (2005).

[Zha] S.-W. Zhang, Gross-Zagier formula for GL(2). II, in: "Heegner Points and Rankin L-series", Math. Sci. Res. Inst. Publ. 49, Cambridge Univ. Press, Cambridge, 2004, pp. 191-214. DOI : 10.1017/CB09780511756375.008.

School of Mathematics, KIAS, Seoul, Korea

E-mail address: chanho.math@gmail.com

Rebut el 20 de desembre de 2017. 